\title{
Hydraulic pitch control system for wind turbines: Advanced modeling and verification of an hydraulic accumulator
}

Irizar, Victor; Andreasen, Casper Schousboe

Published in:

Simulation Modelling Practice and Theory

Link to article, DOI:

10.1016/j.simpat.2017.09.002

Publication date:

2017

Document Version

Peer reviewed version

Link back to DTU Orbit

Citation (APA):

Irizar, V., \& Andreasen, C. S. (2017). Hydraulic pitch control system for wind turbines: Advanced modeling and verification of an hydraulic accumulator. Simulation Modelling Practice and Theory, 79, 1-22.

https://doi.org/10.1016/..simpat.2017.09.002

\section{General rights}

Copyright and moral rights for the publications made accessible in the public portal are retained by the authors and/or other copyright owners and it is a condition of accessing publications that users recognise and abide by the legal requirements associated with these rights.

- Users may download and print one copy of any publication from the public portal for the purpose of private study or research.

- You may not further distribute the material or use it for any profit-making activity or commercial gain

- You may freely distribute the URL identifying the publication in the public portal 


\title{
Hydraulic Pitch Control System for Wind Turbines: Advanced Modelling and Verification of an Hydraulic Accumulator
}

\author{
Victor Irizar ${ }^{1}$ \\ Fritz Schur Energy A/S. Fabriksparken 7, 2600 Glostrup, Denmark \\ Casper Schousboe Andreasen \\ Department of Mechanical Engineering, Solid Mechanics, Technical University of Denmark, Nils Koppels Allé, B.404, 2800 Kgs. Lyngby, \\ Denmark.
}

\begin{abstract}
Hydraulic pitch systems provide robust and reliable control of power and speed of modern wind turbines. During emergency stops, where the pitch of the blades has to be taken to a full stop position to avoid over speed situations, hydraulic accumulators play a crucial role. Their efficiency and capability of providing enough energy to rotate the blades is affected by thermal processes due to the compression and decompression of the gas chamber.

This paper presents an in depth study of the thermodynamical processes involved in an hydraulic accumulator during operation, and how they affect the energy efficiency of the component.

An initial evaluation of the popular thermal time constant model is made and compared with experimental results for a 6 liter accumulator, showing that the current estimation techniques for the thermal time constant are not suited for the application studied, predicting higher heat losses in the gas and resulting in lower pressure buildup. Furthermore, it is shown that the assumption of a constant value for the thermal time constant can provide extremely accurate results, provided that the compression ratios of the process are known in advance. For varying compression ratios, dynamical effects play an important role and the accuracy of the model decreases.

To study the thermal processes, a simplified axisymmetric CFD model of the accumulator is developed. The results show that the main heat transfer losses are associated with heat diffusion in the solid parts of the accumulator, making up to $20 \%$ of the total heat losses. It is also shown that the heat transfer processes and the thermal time constant are tightly connected to variations in gas mass, in rate of change of volume and compression ratios. Comparison with experimental results validate the CFD model accurately, showing high level of agreement and repeatability between the predicted pressures and temperatures and the experimental measurements.
\end{abstract}

Keywords:

CFD, Hydraulics, Accumulators, Heat Transfer, Thermal Time Constant Model

\section{Introduction}

In modern pitch controlled wind turbines, hydraulic systems play an important role regulating their power production, e.g. by controlling the pitch angle of the blades [1]. In general, these systems consist of a hydraulic pump that supplies the oil flow, which is then routed by a set of control valves to a linear actuator attached to the blade (in one or two anchor points on the blade root) and its linear displacement is converted to an angular rotation of the blade. The purpose of the hydraulic control system is to ensure an efficient power production and to protect the turbine from dangerous situations like over-speeding or blade failures. When these emergency situations arise, the hydraulic

\footnotetext{
${ }^{1}$ The work presented in this paper was conducted while being enrolled in the Techical University of Denmark, during the period between January and August of 2015, as a thesis project for the M.Sc. degree in Engineering Design and Applied Mechanics

Email addresses: vie@f senergy.dk (Victor Irizar ), csan@mek.dtu.dk (Casper Schousboe Andreasen)
} 
system has to be able to pitch the blades up to a position where the turbine stops, meaning turning the blades to 90 degrees compared to the wind direction, as fast and reliable as possible. Hydraulic pitch systems include accumulators that store energy during normal operation, in terms of compressed gas ( Figure 1), and when the system performs an emergency stop, the accumulators provide high pressure flow to the actuators so the blade can be turned to the desired stop position.

In an ideal situation, all the energy stored in the accumulator can be utilized and delivered to the hydraulic system without thermal losses at the gas side during compression and expansion, but in reality, the process is not adiabatic. The gas experiences pressure and temperature variations with changes in volume, thus heat is transfered to the solid parts of the accumulator, and consequently to the environment, either through convection, conduction or radiation.

The complexity of these phenomena has been studied and modeled with empirical correlations that provide fairly accurate results for very specific situations $[2,3,4,5]$. Little is reported about what is truly happening in the gas side of the accumulator. A thermal model for accumulators based on heat conduction i.e. an over-all equivalent conduction to all convection contributions were presented in [6] while [7] presented experiments that supports that the heat transfer cannot be described by the average Reynolds number of a cyclic process alone. Further insight into the problem has been reported by [8] who presents numerical studies of a gas spring and the heat transfer during cyclic compression and expansion. Correlations for such cyclic behavior was also the scope of [9] where correlations based on experiments for characterization of the heat transfer in a reciprocating compression-expansion cycle were presented. More recently and in association of the accumulators used for hydraulic systems [10] presented a dynamic model where heat transfer coefficients for the gas-wall interface are table values. The stratified temperature in the gas is not considered, however these effects may increase the local heat transport significantly. A similar type of model were presented in [11] where the heat conduction and convection is modeled similarly but wall temperatures are computed by use of a finite difference model along the outer shell.

For accumulators used in hydraulic systems that posses a non-cyclic behavior there is a need to improve the fidelity of the models of the thermo fluid dynamics behavior i.e. natural convection phenomena, that occurs in the interior of the gas volume. A better understanding of this can help improve current linear models, ultimately leading to optimization on the pitch control systems of the wind turbines, for example by reducing the size or the number of accumulators used per blade.

This paper describes the thermodynamical processes involved in an accumulator in operational conditions in detail and how these are affected by different factors, such as initial pressure and temperatures and compression speeds, in order to setup a simple lumped model for the accumulator. The overall goal is to discuss the accuracy and precision of the actual modeling techniques and how they can be improved. By gaining insight through CFD much more accurate time-constants can be obtained and consequences of e.g. accumulator positioning and direction can be taken into account.

The paper is organized as follows. In Section 2 a brief description of the problem of heat losses in hydraulic accumulators is introduced. Section 3 follows with a deeper explanation and description of the thermal time constant model, a description of the experimental setup used for validation of the models and finishing with an optimization study of the proper time constant to use in the case studied. Section 4 describes the whole CFD modeling and setup. Section 5 presents the validation of the CFD model while Section 6 demonstrates the obtained results, explanations and comparisons with the experimental data. Finally, Section 7 finalizes the work with conclusions on the results and recommendations for future investigations.

\section{Problem Description}

A general layout of a hydraulic accumulator is presented in Figure 1. The oil side has an input port through which the hydraulic oil can flow in or out. A floating piston separates the gas side from the oil. The gas is entrapped in the cylinder and is compressed by the piston when the oil starts flowing into the accumulator.

As the gas is compressed, its temperature and pressure rise. Equivalently, when the hydraulic system demands pressurized oil, the fluid leaves the accumulator and the gas expands, decreasing both its temperature and pressure. Heat transfer occurs between the gas and the solid parts of the accumulator, through convective and conductive mechanisms. The rate at which the heat transfer occurs is dependent on factors like the properties of the gas, pressure, temperature, initial conditions and the speed of the volume changes. 


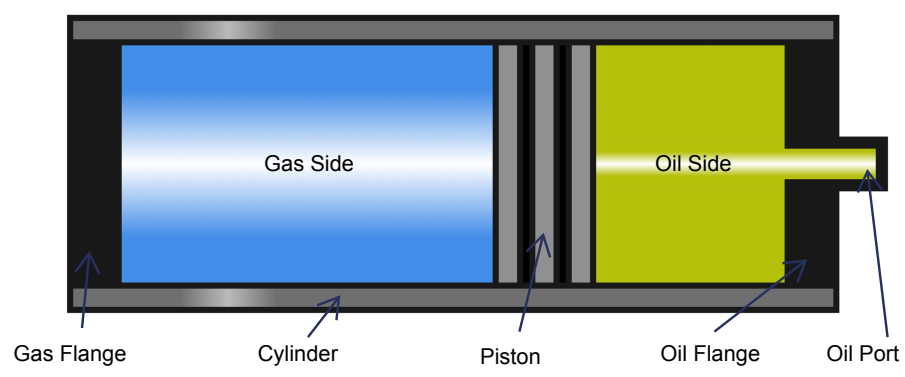

Figure 1: Basic layout of a piston type hydraulic accumulator

For transient simulations, the mathematical modeling of a gas charged accumulator is often based on the thermal time constant model $[2,12,3]$. The accuracy of the model relies on the selection of an appropriate value for the time constant, for which several correlations or guidelines exist depending on the type of accumulator [5, 4], but often these correlations are not generalizable and fail to be consistent for different types of accumulators and working conditions.

\section{Revisiting the Thermal Time Constant Model}

\subsection{Energy Balance on the Gas Side}

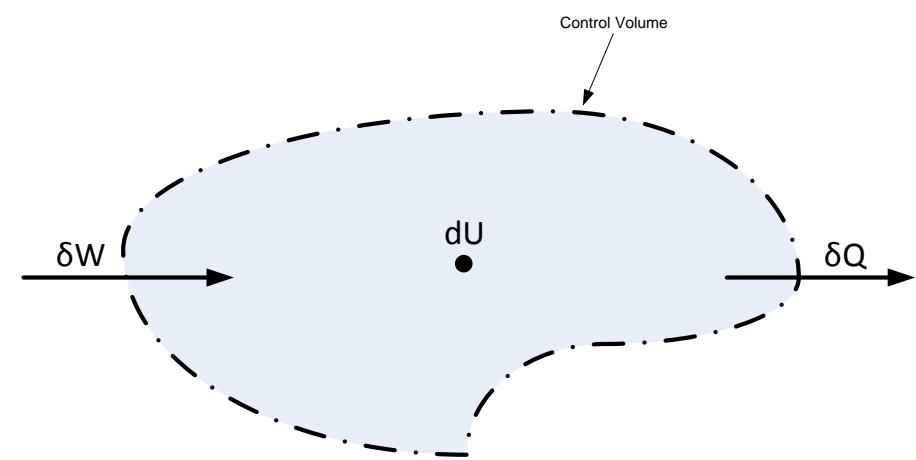

Figure 2: First law of thermodynamics (Conservation of Energy) for a control mass over an instant of time

The energy balance over an arbitrary control volume, where no material losses or leakages out of the domain are experienced, as shown in Figure 2, is mathematically expressed as

$$
d U=\delta Q+\delta W
$$

which states that the change of the internal energy of the system dU is equivalent to the heat exchange between the control volume and the surroundings $\delta Q$ minus the work done by the control volume on its surroundings $\delta W$.

For a real gas, the change of internal energy due to the changes in temperature and pressure is given by

$$
d U=m C_{V} d T+\left[T\left(\frac{\partial p}{\partial T}\right)_{V}-p\right] d V
$$

where $m$ is the gas mass, $C_{V}$ is the specific heat capacity at constant volume of the gas, $T$ is the bulk temperature of the gas, $p$ is the pressure and $V$ is the volume of gas. The work done by the gas due to pressure and volume changes is defined as

$$
\delta W=-p d V
$$


With convection as the leading mechanism of heat transfer from the gas to the solid parts of the accumulator, the heat term can be expressed as

$$
\delta Q=\oint h\left(T_{w}-T\right) d A
$$

where $h$ is the heat transfer coefficient (which is a function, often non linear, of the temperatures, pressures, and velocity of the gas), and $T_{w}$ is the temperature of the wall, and the heat exchange is the surface integral of the boundary heat flux $h\left(T_{w}-T\right)$ over the area $d A$. Inserting (2), (4) and (3) in (1), results in

$$
m C_{V} \frac{d T}{d t}+\left[T\left(\frac{\partial p}{\partial T}\right)_{V}-p\right] \frac{d V}{d t}=\oint h\left(T_{w}-T\right) d A-p \frac{d V}{d t}
$$

which represents the main energy balance in the gas domain. To fully define the temperature and pressure of the domain at any time, an equation of state (EOS) of the gas is needed. In general, the equation of state relates pressure, temperature and volume of the gas satisfying the equality

$$
f(T, p, V)=0
$$

Many equations of state have been developed and implemented efficiently for various real gas applications. Here the Soave-Redlich-Kwong (SRK) is chosen, which has been shown to be accurate and computationally efficient by [1] for modeling hydraulic accumulators.

The SRK equation of state defines the gas pressure as a function of the temperature and molar volume as

$$
p=\frac{R T}{V_{m}-b}-\frac{a \alpha}{V_{m}\left(V_{m}+b\right)}
$$

where $R=8.314 \mathrm{~kJ} / \mathrm{kgK}$ is the universal gas constant, $T$ is the gas temperature, $V_{m}$ is the molar volume of the gas (which is the ratio between the molar mass of the gas $M$ and its density $\rho$ ), $b, a$ and $\alpha$ are gas specific parameters defined as

$$
\begin{aligned}
a & =\frac{0.427 R^{2} T_{c}^{2}}{P_{c}} \\
b & =\frac{0.0866 R T_{c}}{P_{c}} \\
\alpha & =\left(1+\left(0.48+1.574 \omega-0.176 \omega^{2}\right)\left(1-T_{r}^{0.5}\right)\right)^{2}
\end{aligned}
$$

where $T_{c}$ and $P_{c}$ are the gas critical temperature and pressure, $\omega$ is the accentric factor of the gas, and $T_{r}=T / T_{c}$ is the relative temperature. For the studies presented here, the properties used for diatomic nitrogen $N_{2}$ are: $T_{c}=$ $126.2 \mathrm{~K}, P_{c}=33900000 \mathrm{~Pa}, \mathrm{M}=0.028 \mathrm{~kg} / \mathrm{mol}$ and $\omega=0.04$.

The thermodynamical balance of energy in the gas domain of the accumulator is represented by (5). Assuming that there is an overall convective phenomena represented by a single heat transfer coefficient $h$, and that the boundaries of the domain are all at a fixed ambient temperature $T_{a}$, the balance can be re written as

$$
m C_{V} \frac{d T}{d t}+\left[T\left(\frac{\partial p}{\partial T}\right)_{V}-p\right] \frac{d V}{d t}=h A\left(T_{a}-T\right)-p \frac{d V}{d t}
$$

rearranging the terms and isolating the rate of change of the temperature of the gas domain, the balance reduces to

$$
\frac{d T}{d t}=\frac{T_{a}-T}{\tau}-\frac{T}{C_{V}}\left(\frac{\partial p}{\partial T}\right)_{V} \frac{d v}{d t}
$$

where $v=\frac{V}{m}$ is the specific mass of the gas, and the thermal time constant $\tau$ is defined

$$
\tau=\frac{m C_{V}}{h A}
$$

This model was initially proposed by Otis [2], and coupled with the Soave-Redlich-Kwong EOS, (work done by [1]). The evolution of both the temperature and pressure of the gas domain can be predicted, given that a proper expression of the thermal time constant $\tau$ is provided. 


\subsection{Interpretation of the Thermal Time Constant}

The parameter $\tau$ then relates the thermal capacitance of the system with the convective heat losses to the walls due to the temperature changes in the domain. The higher the thermal time constant, the closer the system will behave to an adiabatic system. If the convective heat losses are very high or the mass of the system is relatively low (expected for gases), the thermal time constant becomes lower, contributing to the decay of both pressure and temperature. Experimentally, the thermal time constant has been calculated by observing the pressure decay in the gas due to a step response in the gas volume [13]. After the gas volume has been compressed, during the constant volume pressure relaxation, the gas temperature can be described as

$$
\frac{d T}{d t}=\frac{T_{a}-T}{\tau}
$$

which corresponds to an exponential decay with time constant $\tau$. This represents the time that the gas takes to drop its temperature (or temperature) by 63.2 percent. Based on this experimental procedure, different correlations have been developed to predict the magnitude of the thermal time constant as a function of parameters like the initial temperature and pressure of the gas as well as the size of the accumulator $[4,5,13]$.

\subsection{Comparison with Experimental Results}

In order to asses the accuracy of the thermal time constant model, an experimental validation was carried out. The experimental procedure and test equipment used in [1] served as a base for the validation carried out in this work. The gas temperature and pressure measured on a 6 liter accumulator subjected to compression-expansion processes were compared to the predictions of the thermal time constant model coupled with the RKS equation of state and a correlation developed by [4] to calculate the value of the thermal time constant parameter. This correlation is defined by the following equation for piston type accumulators

$$
\tau=\frac{293}{T_{0}}\left(0.3 p_{0} V_{0}^{0.33}+86.2 V_{0}^{0.49}\right)
$$

where the thermal time constant is a function of the initial volume $V_{0}\left[\mathrm{~m}^{3}\right]$, temperature $T_{0}[K]$ and pressure $p_{0}[b a r]$ of the accumulator. This correlation is semi-empirical and shows good agreement with experimental data from [5] in the work done by [4].

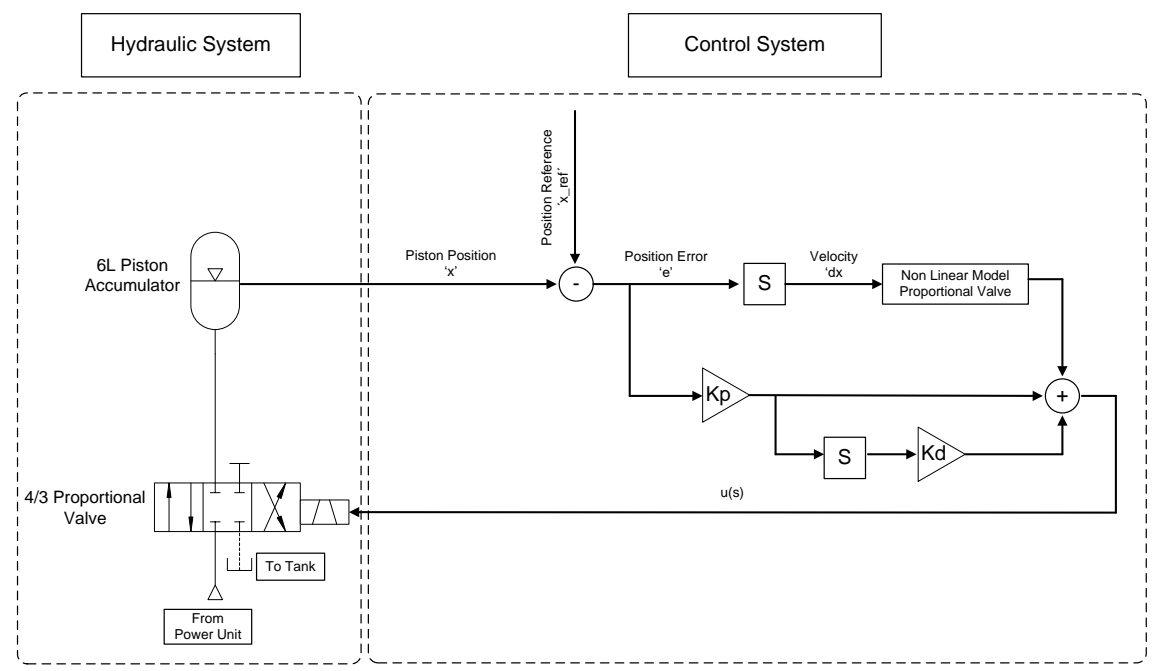

Figure 3: Hydraulic diagram of the experimental setup used for validation

A diagram of the experimental setup used can be seen in Figure 3, with pictures of the main components presented in Figure 4. The experimental setup consists of a test accumulator, mounted in a pivoting support to allow orientation changes (with the possibility to be rotated by 180 degrees from a vertical position), shown in Figure 4a. Oil is supplied 
to the test rig by an hydraulic power supply composed of two pumps and 4 accumulators (Figure $4 \mathrm{~b}$ ). The supply is capable of delivering hydraulic oil at a maximum pressure of 250 bar.

The oil flow into the accumulator is controlled by a $4 / 3$ proportional valve. The position of the piston is controlled via a P-I controller, which receives the position signal of the piston and adjusts the flow through the hydraulic valve accordingly, using also a feed-forward loop on the velocity of the piston.

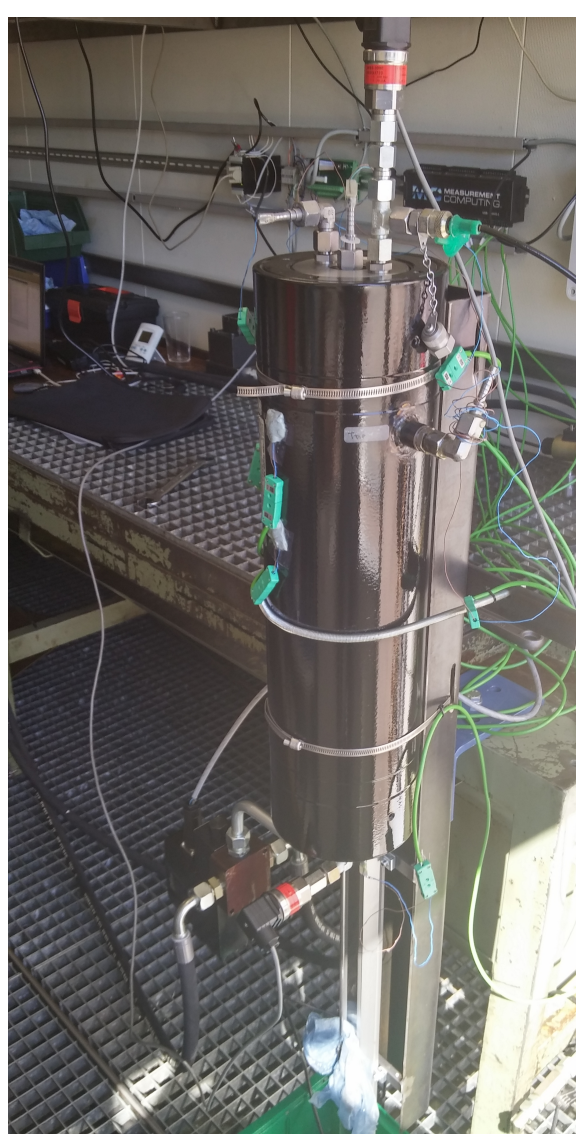

(a) Test Accumulator

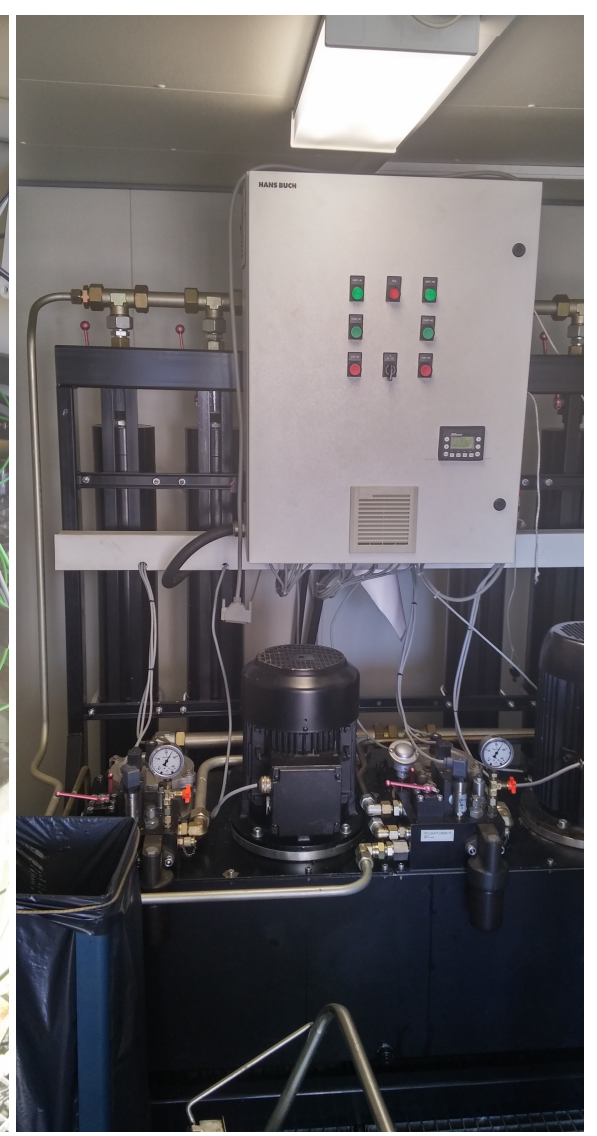

(b) Hydraulic Supply

Figure 4: Main hydraulic components of the experimental rig at FSE

The test accumulator is connected to a variety of sensors to monitor pressure and temperature at different points. Figure 5 shows the sensor positioning in the accumulator, corresponding to the schematic shown in Figure 6 . The gas chamber is connected to a 400 bar pressure transmitter. In addition to that, two thermocouples are positioned in the gas side, one in the center of the piston and the other on the top flange. The thermocouples are exposed welded tip K type thermo couples from RS components with a measured time constant of $7 \mathrm{~ms}$. Four more probes monitor the outer shell temperature (only two shown in the figure, two extra sensors are positioned in the same axial coordinates but with a radial offset of 90 degrees). The piston is attached to a rod that drives a resistive position sensor, which lies outside of the accumulator. All the sensors are connected to a data acquisition system by Measurement Computing which is itself linked to a main computer equipped with DASYLab software. This software enables the creation of data acquisition interfaces, and handles the input-output and control processes. A data acquisition layout was developed where a reference signal of the position of the piston in time could be introduced as a text file and the program returns the sensor readings over time, also as a text file.

The accumulator is subjected to compression-expansion cycles where the initial temperature, pre-charge and final pressures where varied, based on the experiments of [1]. A three digit labeling system for the experimental runs is used to identify the different levels at which the above mentioned conditions can vary, following Table 1, for a total 


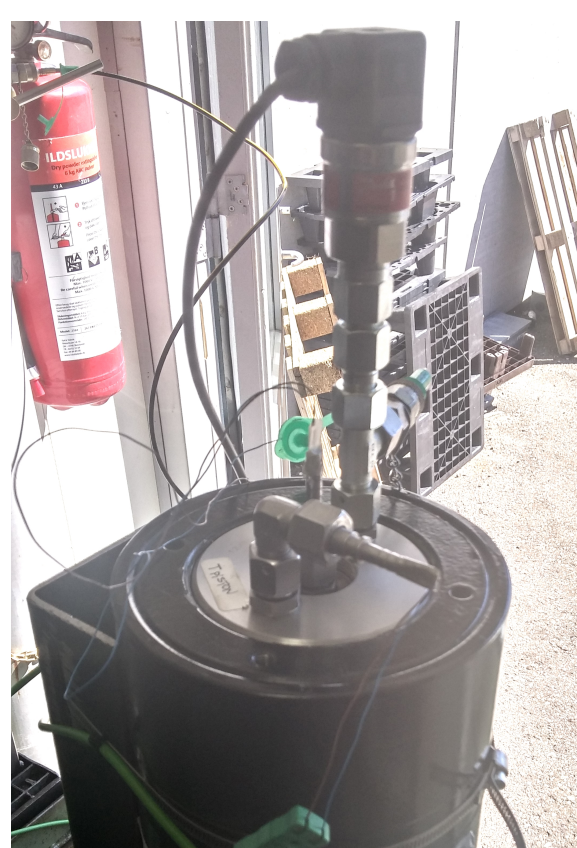

(a) Pressure and Temperature Sensors Top

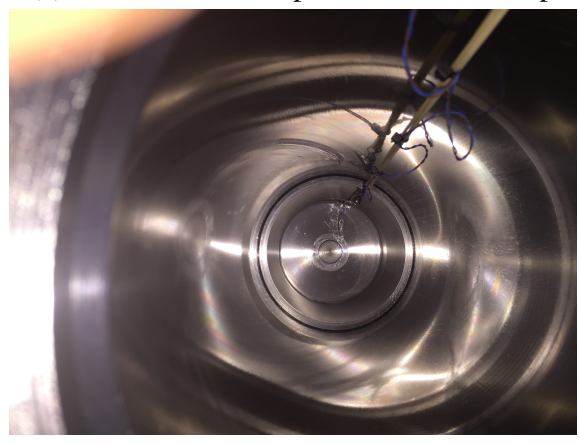

(c) Temperature Sensor in Piston

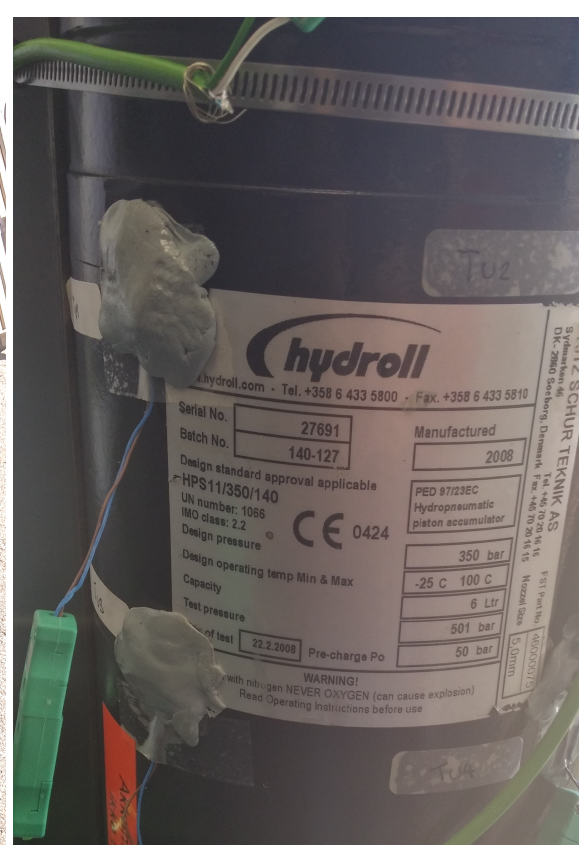

(b) Outer Shell Temperature Sensors

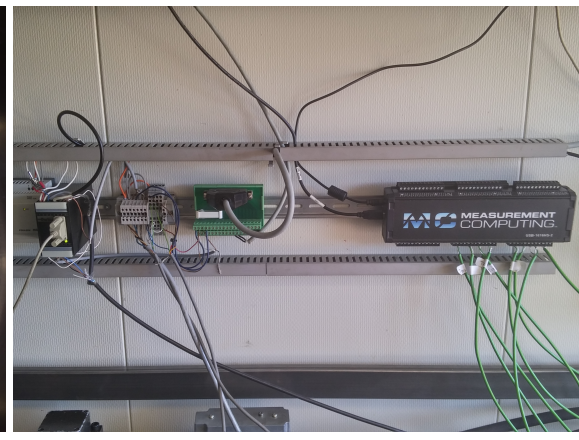

(d) Data Acquisition Modules

Figure 5: Temperature and pressure sensors and data acquisition front end in experimental rig

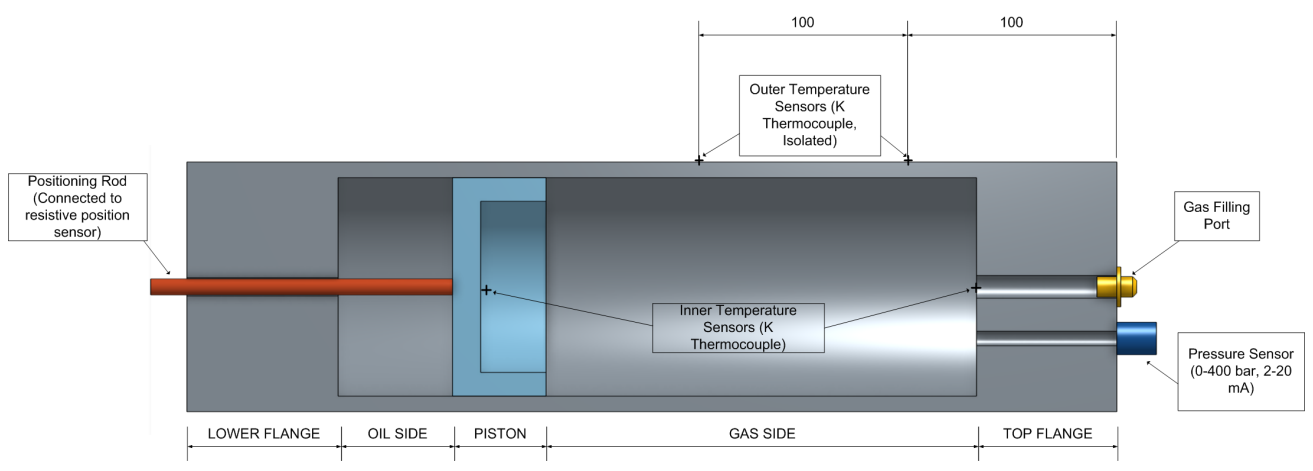

Figure 6: Basic schematic of sensor positioning in the test accumulator. Lengths in $\mathrm{mm}$.

of 27 experimental cycles. The volume of the gas side followed the shape of the curve presented in Figure 7, where an initial steady maximum volume is held for an amount of time, followed by a compression phase, with a volumetric 
speed of $0.53 \mathrm{~L} / \mathrm{s}$ (linear piston velocity of $33 \mathrm{~mm} / \mathrm{s}$ ), after which the minimum volume is also sustained for an specific amount of time, finalizing with a decompression phase, with an average volumetric speed of $0.41 \mathrm{~L} / \mathrm{s}$ (linear piston velocity of $25 \mathrm{~mm} / \mathrm{s}$ ). For these tests, the accumulator was oriented horizontally.

\begin{tabular}{ccc}
\hline$T_{0}\left[{ }^{\circ} \mathrm{C}\right]$ & $p_{0}[\mathrm{bar}]$ & $p_{\text {end }}[\mathrm{bar}]$ \\
\hline-20 & {$[50,100,150]$} & {$[180,220,250]$} \\
20 & {$[50,100,150]$} & {$[180,220,250]$} \\
60 & {$[50,100,150]$} & {$[180,220,250]$} \\
\hline
\end{tabular}

Table 1: Experimental setups for the tests. A three digit labeling system for the experimental runs is used to identify the different levels at which the above mentioned conditions can vary (ex. run $311=$ Initial Temperature 3, Precharge Pressure 1, Final Compression Pressure Level 1).

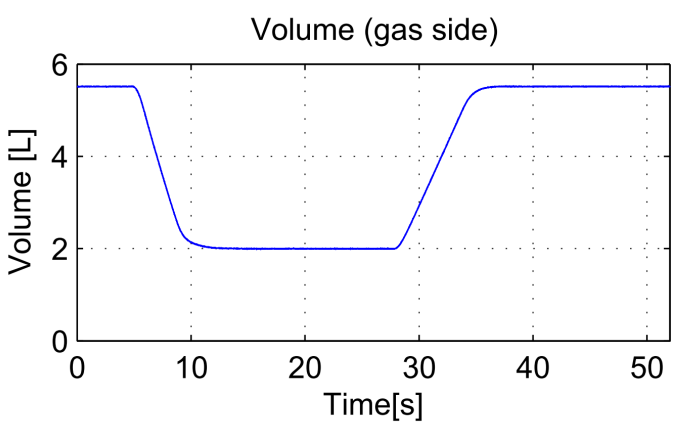

Figure 7: Gas side volume as a function of time

Figure 8 shows the comparison between the experimental results and the simulated thermal time constant model for two selected cases, which represent the most and least accurate predictions from the model. The value of $\tau$ used for the modeled results was calculated using Eq. 15. For both cases, the modeled pressures and temperatures are under predicted after the end of the compression phase, evidencing that the $\tau$ constant used in the model was predicted lower than the real time constant of the system. Nevertheless, it can be seen in the pressure plots (Figures $8 \mathrm{a}$ and $8 \mathrm{c}$ ) that the simulated decay after the compression has a similar behavior than the one seen in the experimental results. According to Otis and Pourmovahed[13] the system should behave as a first order system, with a decay governed by a time constant, however as this can be difficult to confirm from Figures 8 this is confirmed by a longer cycle shown in Appendix A. The discrepancies between experimental and simulated results hold true also for the gas temperature ${ }^{3}$ (Figures $8 \mathrm{~b}$ and $8 \mathrm{~d}$ ). Despite the discrepancies after the compression phase, the simulated pressure follows the experimental one accurately in the rest of the cycle, which suggest that the chosen $\tau$ has small influence in the expansion phase, compared to how it affects the compression. Examining the relative errors between all the experimental and the simulated results obtained, the average root mean-square (abbreviated RMS) error sits at $6 \%$ for both temperature and pressure and the maximum errors reach up to $16 \%$ for the pressure and $18 \%$ for the temperature. This discrepancies suggest that the $\tau$ presented by [4] is not extensible to this accumulator because it over-predicts heat losses in the gas.

\subsection{Finding the Proper Thermal Time Constant}

Observing the predictions of the thermal time constant model compared to the experimental results, it is plausible that there exist a value of $\tau$ that would make the model predictions to fit the experimental measurements. An approach

\footnotetext{
${ }^{3}$ The temperature comparisons need to be interpreted with care since the positioning of the temperature probes in the experimental setup can give temperature values that do not resemble the overall gas temperature due to gradients inside of the gas. Static pressure comparisons are reliable since pressure gradients are negligible for low speed flows $(M a<0.3)$.
} 


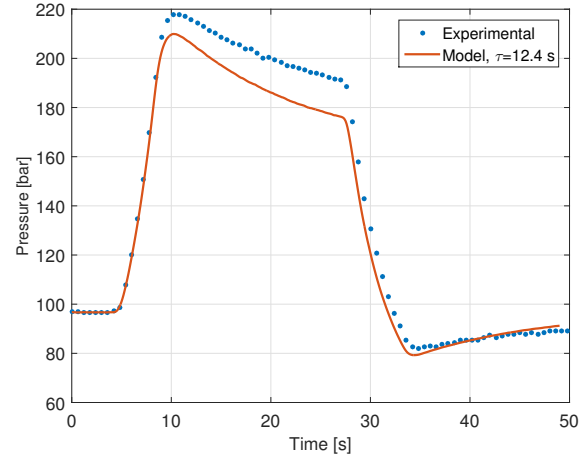

(a) Gas Pressure, Test Run 222

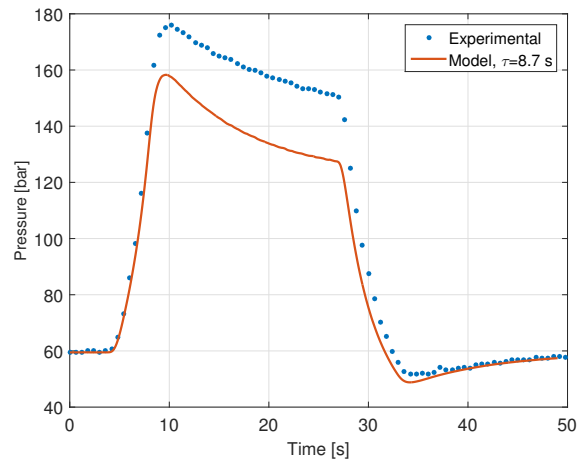

(c) Gas Pressure, Test Run 311.

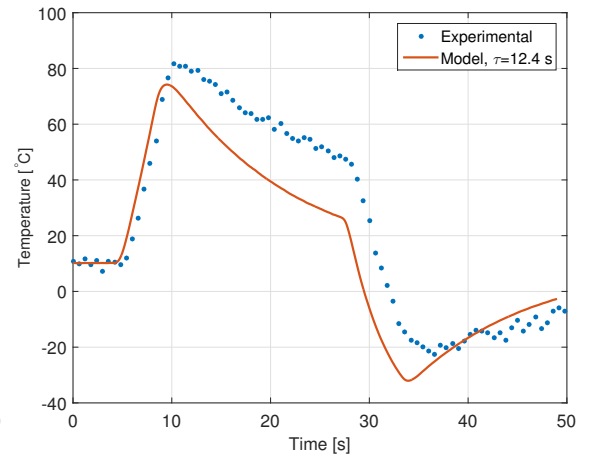

(b) Gas Temperature, Test Run 222.

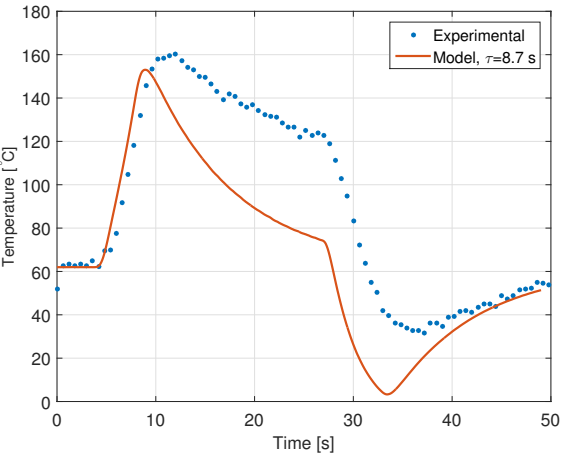

(d) Gas Temperature, Test Run 311.

Figure 8: Comparison between experimental and results from thermal time constant model for selected test runs. Run 222 specifications: $T_{0}=$ $10.2^{\circ} \mathrm{C}, p_{0}=96.7 \mathrm{bar}, p_{\max }=209.9 \mathrm{bar}$. Run 311 specifications: $T_{0}=61.9^{\circ} \mathrm{C}, p_{0}=59.5 \mathrm{bar}, p_{\max }=158.3 \mathrm{bar}$.

to increase the precision of the model is to identify $\tau$ by solving a least squares minimization problem as

$$
\begin{array}{ll}
\underset{\tau \in \mathfrak{R}}{\operatorname{minimize}} & \varepsilon=\left(p_{\text {exp }}-p_{\text {model }}(\tau)\right)^{2} \\
\text { subject to } & 1<\tau \leq 300
\end{array}
$$

where $\varepsilon$ is the error between the experimental pressure $p_{\text {exp }}$ and the calculated pressure using the thermal time constant model $p_{\text {model }}(\tau)$, using as inputs the guess value of the thermal time constant $\tau$, the initial conditions and the rate of change of volume extracted from the experimental results. $\tau$ is constrained between 1 (representing an almost instantaneous heat transfer to the boundaries) and 300 (large enough value that represents an adiabatic system). The thermal time constant was obtained by applying non linear regression, using the damped least squares method (Levenberg-Marquardt algorithm [14]), which is implemented in MATLAB through the function 'nlinfit'.

Figure 9 shows the results comparison between the experimental results, the modeled temperatures and pressures using the thermal time constant model with $\tau$ calculated with Eq. 15 and the ones with the optimized $\tau$. It can be seen that the agreement between the optimized results and the experimental ones has improved compared to the original model. The RMS relative errors of the pressure prediction now have dropped to an average of $1.5 \%$, with maximum relative errors below $8 \%$. The time constants calculated with the optimization are on average 2.7 times higher than the ones predicted with Eq. 15, reaching in some up to 4 times higher values. These results support the assumptions that a constant value of $\tau$ is able to model the real heat transfer processes.

In Figure 10 the influence of the initial pressure, initial temperature, gas mass and compression ratio on the optimized $\tau$ constant calculated through the minimization problem is presented. It can be noticed that $\tau$ has a clear tendency to increase as the initial pressure raises (Figure 10b). This is a direct consequence of the fact that increased pressures translate to a higher amount of gas mass being enclosed in the accumulator, and therefore, by Eq. 13, the thermal time constant increases proportionally (Figure 10c). Similarly, lower temperatures imply higher densities and 


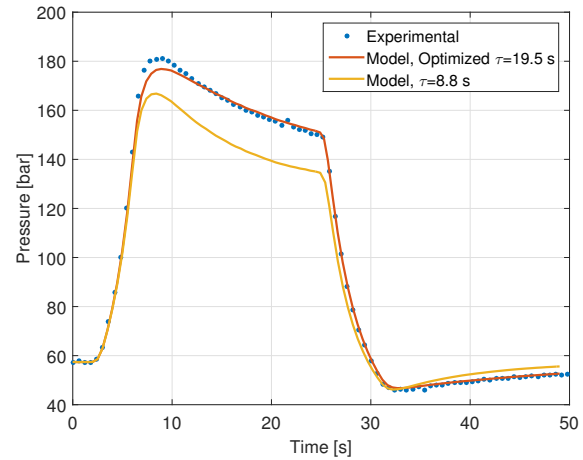

(a) Gas Pressure, Run 111.

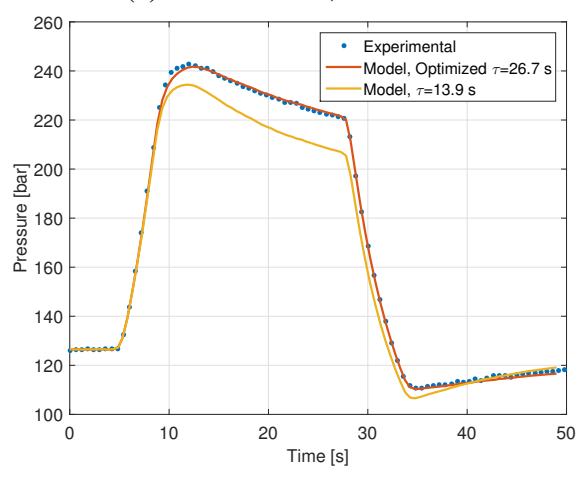

(c) Gas Pressure, Test Run 233 .

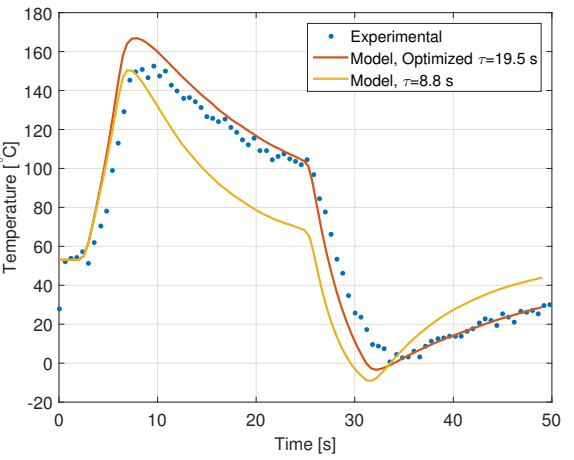

(b) Gas Temperature, Test Run 111.

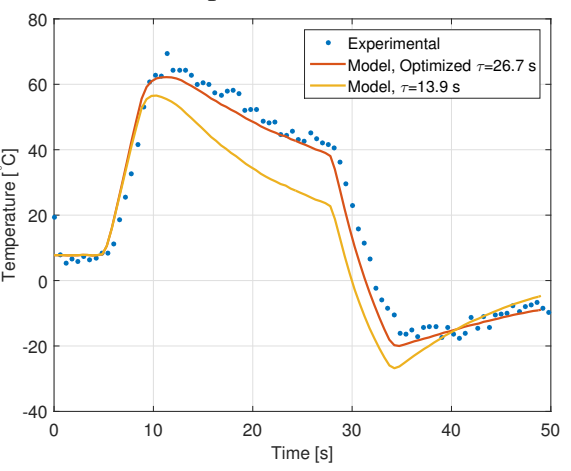

(d) Gas Temperature, Test Run 233.

Figure 9: Comparison between experimental and simulated results from thermal time constant model for selected test runs, based on the study of [1], and results for the optimized thermal time constant. Run 111 specifications: $T_{0}=52.3^{\circ} \mathrm{C}, p_{0}=57.4 \mathrm{bar}, p_{\max }=166.7 \mathrm{bar}$. Run 233 specifications: $T_{0}=8.1^{\circ} \mathrm{C}, p_{0}=126.5 \mathrm{bar}, p_{\max }=234.1 \mathrm{bar}$.

increased gas mass. However, no clear dependency of the thermal time constant to the initial temperature can be seen in Figure 10a, which can be attributed to the difficulties presented in measuring accurately the temperature of the gas in the chamber, as well as the difficulties in heating and cooling uniformly the cylinder and the gas.

The most interesting relationship can be found in Figure 10d, since it reflects that the thermal time constant is dependent on the compression ratio of the process. At high compression ratios, more energy is internally generated in the gas and higher temperatures and pressures are developed, which increases the heat transfer to the surroundings. At lower compression ratios temperatures and pressures in the gas domain reach lower levels and also less momentum is transfered to the fluid due to the reduced piston movement, consequently decreasing the heat losses.

This dependency of the thermal time constant to the compression ratios of the gas domain implies that a constant $\tau$ can provide a very good approximation to the heat transfer in the gas, if the compression ratios are known beforehand. But for a process where the accumulator is subjected to variable non uniform compression-expansion processes, a constant $\tau$ will not be able to resemble the dynamics of heat transfer.

\section{CFD Modeling}

The main objective of the modeling is to develop a simple geometrical representation of the accumulator, that takes into account the main features of the real component and provides accurate results, comparable to the real life behavior of the device.

In order to study in depth the thermodynamics of the gas in the accumulator, a CFD model was developed. For the modeling of the accumulator, the use of two softwares was implemented. For the CAD modeling and meshing, COMSOL Multiphysics was used due to its powerful and flexible mesher and STAR CCM+ was then used for solving and post-processing of the results. 


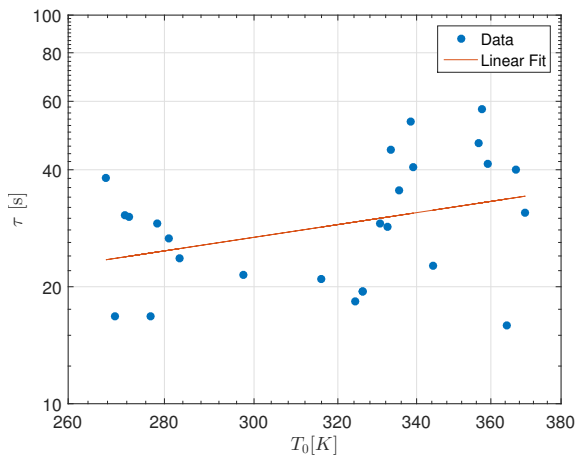

(a) $\tau$ vs. $T_{0}$

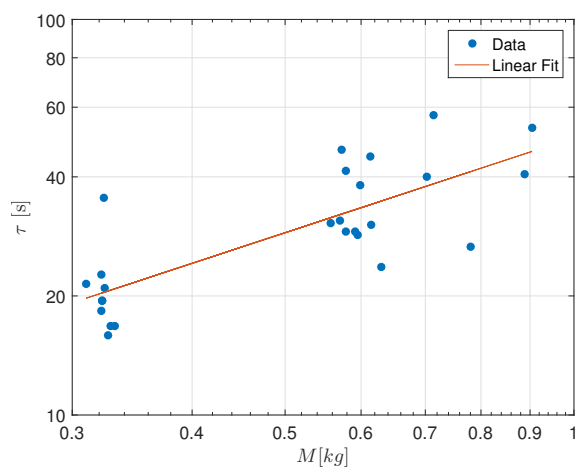

(c) $\tau$ vs. $m$

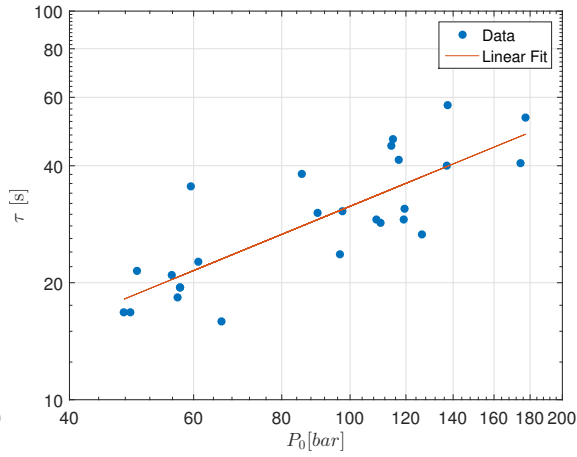

(b) $\tau$ vs. $P_{0}$

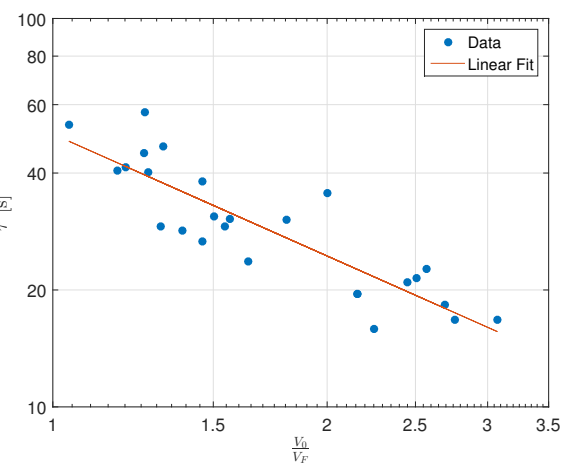

(d) $\tau$ vs. $V_{0} / V_{F}$

Figure 10: Dependency of the thermal time constant to different parameters.

\subsection{Geometry}

The accumulator has the advantage of being of a symmetrical cylindrical shape, so to minimize the computational effort, a three dimensional wedge of the cylindrical domain was modeled, to be able to exploit the axis-symmetrical properties of the cylindrical shape of the accumulator. This wedge has an angular length of four degrees as seen in Figure 11.

The main geometry is divided in 5 sub domains which are grouped by the type of physics modeling involved in them. Grey parts are solid sections made of steel which are the piston, cylindrical shell and top flange. The yellow domains are the oil side parts, which are the inlet port and the oil cavity. Finally the blue domain represents the gas chamber. The dimensions of the model are taken from CAD models provided by the accumulator manufacturer.

\subsection{Assumptions}

Many geometrical details were not included to make the domain as simple as possible and avoid discretization and numerical errors in places where small cavities or sharp corners might exist. The original piston shape was simplified to a plain cylindrical one, upper and lower flange details like threads and clearances with other parts were ignored and represented as smooth surfaces without gaps.

The accumulator interacts with the outer environment by transmitting convective heat to the surroundings. To economize computational time, no fluid domain was modeled for the ambient air, instead a convective boundary condition of the form $Q_{\text {ambient }}=h_{\text {air }}\left(T_{\text {wall }}-T_{\text {ambient }}\right)$ was set on all the outer walls of the accumulator, where $T_{\text {ambient }}$ is a known measurable parameter from the experiments and $h_{\text {air }}$ is the convective heat transfer coefficient of stall air, which is set to $10 \mathrm{~W} / \mathrm{m} \cdot \mathrm{K}$ based on [15]

It is assumed that during the compression-expansion procedure in the accumulator, the outer walls of the cylinder remain fairly close to the initial conditions and since the difference with the outer temperature is relatively small 


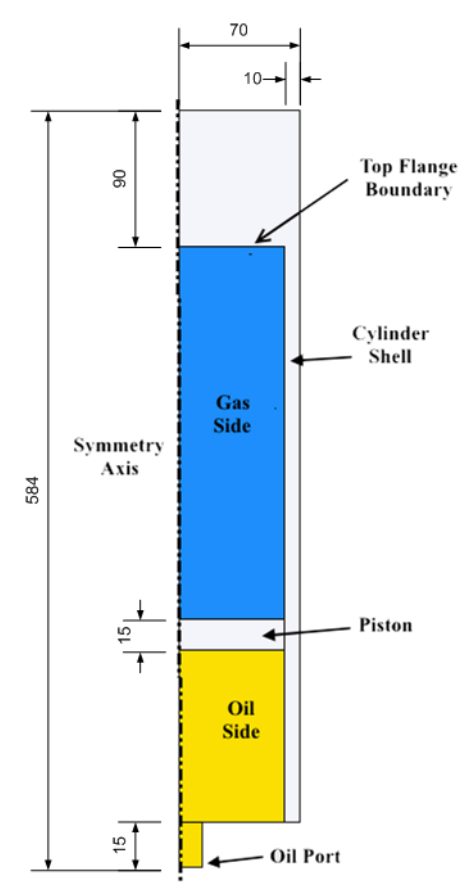

(a) Front View. Measurements in mm.

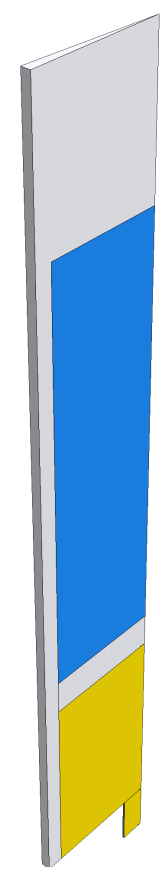

(b) Isometric View

Figure 11: CAD Model of the accumulator with differentiated colored domains (yellow for oil, blue for gas and grey for steel).

(below $40^{\circ} \mathrm{C}$ ), the contribution of the radiation to the heat transfer remains fairly small compared to the convective heat transfer, and can be safely neglected [15].

Compression and expansion processes for the accumulator in a real life situation on a wind turbine occur at piston velocities between $20 \mathrm{~mm} / \mathrm{s}$ and $100 \mathrm{~mm} / \mathrm{s}$, and taking into account that the viscosity of the gas is fairly low compared to any liquid, the influence of the inertial forces in the domain is important and turbulent behavior can be expected. Also, due to the high temperature differences reached between the gas domain and its surroundings after compression or expansion, free convection effects due to buoyancy forces are relevant and consequently turbulent.

The oil domain is included in the simulation since the convective effects of the fluid are considered relevant for the overall heat transmission in the solid parts.

\subsection{Solver Setup}

The transient simulation is solved by a fixed step implicit solver, with a time step of $0.001 \mathrm{~s}$, and first order discretization in time. The inner solver steps are performed in a segregated manner, where the momentum, pressure, energy and turbulence equations are solved one after the other. The inner solver loops until the residuals of the main quantities fall below a predefined tolerance (below $10^{-4}$ ). The differential equations are solved by a multi-grid method.

\subsection{Regions and Domains}

The model contains 3 main physical regions, corresponding to the 3 different material domains that can be found in the accumulator: Liquid for the oil domains, Gas for the accumulator's gas chamber and Solid regions for the remaining steel parts of the accumulator. Each region has an associated set of physical models selected, depending on the simulation needs.

In the gas region, the material used is diatomic Nitrogen $N_{2}$, which is built into the software's material database. Viscosity, thermal conductivity and specific heat capacity are considered functions of pressure and temperature. Force of gravity is enabled in the direction negative of the symmetry axis of the model. The turbulence model $k-\epsilon$ is chosen based on computational efficiency and high accuracy for fully turbulent flows. A turbulence intensity of $4.5 \%$ and a turbulent viscosity ratio of 25, based on [16]. The real gas equation of state used is Soave-Redlich-Kwong. 
In the liquid region, material used is Hydraulic Oil based on the specifications of Castrol AWHM 32 [17] . Viscosity, thermal conductivity and specific heat capacity are considered constant. Gravity force is neglected and the flow is considered laminar since it is a high viscosity fluid. The oil is also considered isothermal.

In the solid region, material used is Carbon Steel based on the specifications of the manufacturer of the accumulator. Constant thermal conductivity of $k=49.8 \mathrm{~W} / \mathrm{mK}$ and specific heat capacity of $C_{p}=4900 \mathrm{~J} / \mathrm{kgK}$ were chosen.

\subsection{Mesh}

Based on the simplicity of the geometrical model, a mapped mesh with quadrilateral elements was chosen for the fluid domains, and a free triangular mesh was chosen for the rest of the solid parts of the geometry, which adapts to parameters selected for the fluid domains. These parameters control the number of elements present in the gas and oil domains to achieve the highest quality possible during the deformation and displacements of the domains. For accurate turbulence modeling, 10 boundary layers were added to the inner side of the boundaries of the gas domain.

The mesh can be seen in Figure 12. In the gas domain the structured mesh can be clearly seen and the boundary layers are properly defined (Figure 12b). In the solid domains, the free tetrahedral mesh can be seen covering the domain. In Figure 12c an area of high density tetrahedral elements can be seen, which covers the side of the cylinder shell that coincides with the fluid domain. This is due to the way the deforming mesh is generated and how it affects the generation of the solid tetrahedral mesh. The oil domain mesh at the beginning of the simulation is compressed, and the elements have a very high aspect ratio. When the oil and gas domain meshes are generated, the final meshing step generates the tetrahedral mesh, using the already generated nodes of the other meshes as guidance. Therefore this generates a very high density tetrahedral mesh in places where the oil or gas meshes have also high density of nodes. This is a limitation of the meshing software used. An alternative more efficient way would be to generate 3 separate meshes and use interpolated interfaces for the transported quantities (heat) in the walls.

The mesh size and setup was optimized to provide the highest accuracy and smallest level of distortion in the gas side mesh. The mesh deformation compresses and stretches the elements of the gas and oil domains, but there is no re-meshing step in between the solving steps.

\subsection{Calculation of Pressure and Temperature}

The CFD model includes a different set of probes and sensors to calculate the relevant properties and be able to truly compare with the real measurements in the experimental setup. The gas pressure is calculated as a mass average of the pressure in every cell. The average temperature is also calculated as a mass average of the individual temperature of all the cells. In addition to that, temperature probes were set in the model following the drawing in Figure 6. 


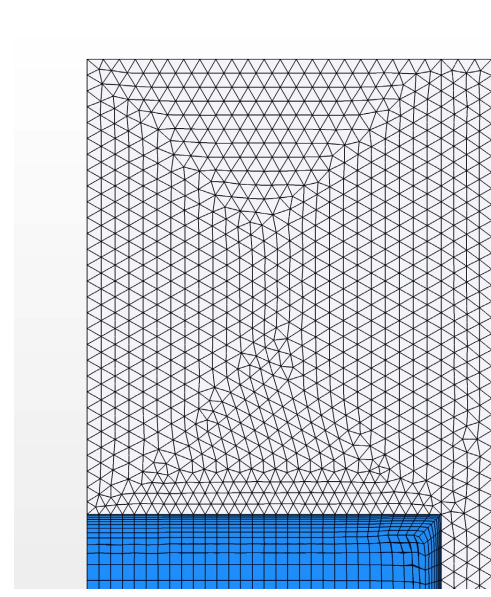

(a) Gas and Top Flange

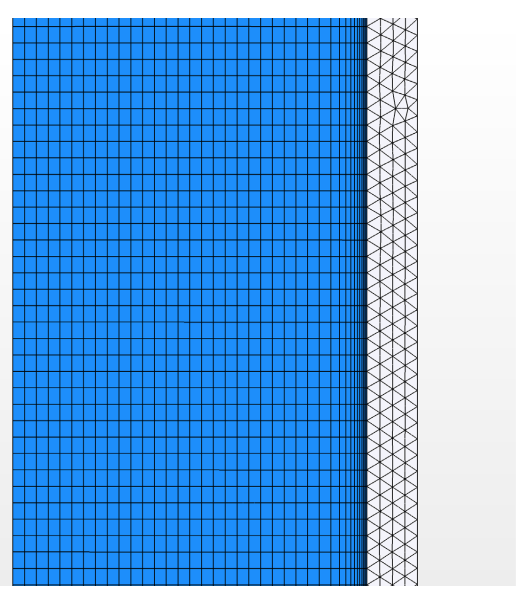

(b) Gas and Cylinder Shell

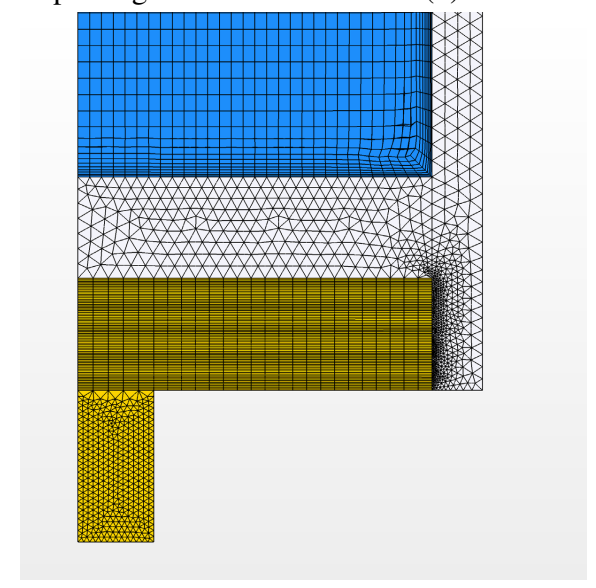

(c) Oil, Piston, Gas and Cylinder Shell

Figure 12: Detail of the model's mesh. Final iteration, number of elements: 4235.

\section{CFD Model Validation}

In order to ensure that the model represents the real component, subject to real life conditions, a validation was carried out using the experimental runs described in Table 1. The data obtained from this test runs includes the piston position over time and temperature and pressure of the gas domain. The validation was carried out with data from 19 selected runs from the previous study.

Figure 13 shows the validation results for selected experimental runs. It can be seen that the simulated results obtained with the CFD model, visually fit accurately the experimental curves. Figure 13e shows the worst case scenario for validation, where the maximum pressures are not adequately reached, but still the decay in the pressure for the simulated result resembles the experimental accurately. Figure 13a and Figure 13c show results were the simulated data matches with higher degree of accuracy the experiments. In general, the average maximum error in pressure between the experimental and simulated results is of $5 \%$, and the average overall RMS error is of $2 \%$, which demonstrates that the model generates repeatable and reliable results for the range of conditions used in the previous experimental studies. The source of the errors that can be seen between the experimental and the simulated data can be explained by different factors. First, the reduced nature of the model, which neglects geometrical features and assumes symmetric flows (no swirl). Second, the uncertainties of experimental data, which include the noise in the signals, the reliability of the sensor positioning and the filtering techniques. And third, the numerical errors introduced by the deformation of the mesh in the CFD model and the degraded cell quality in some regions. 


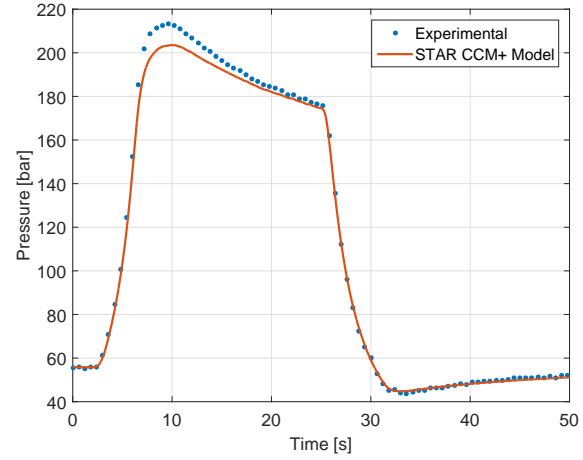

(a) Gas Pressure, Run 112

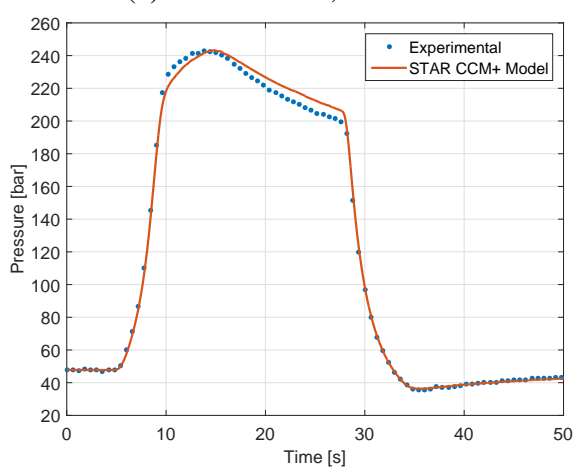

(c) Gas Pressure, Run 213

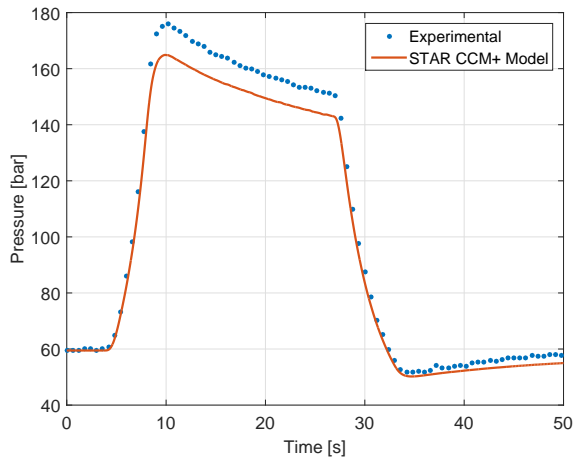

(e) Gas Pressure, Run 311

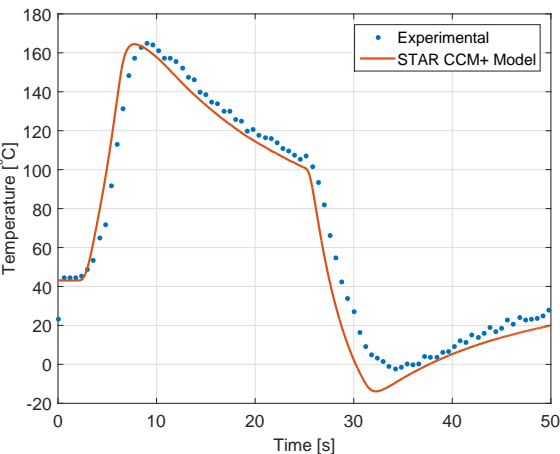

(b) Gas Temperature, Run 112

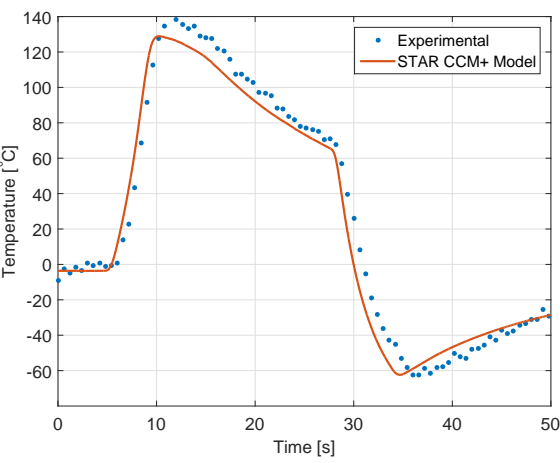

(d) Gas Temperature, Run 213

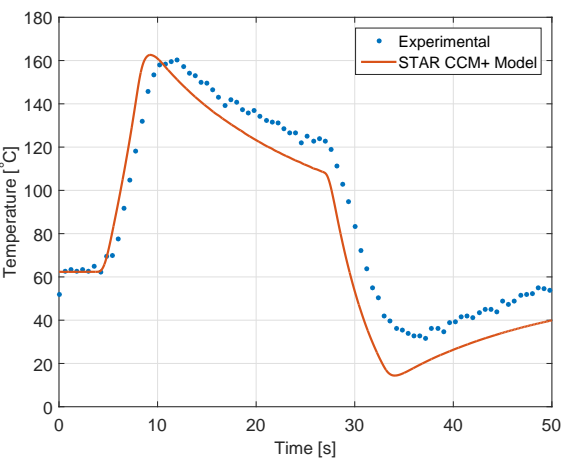

(f) Gas Temperature, Run 311

Figure 13: Comparison between experimental curves from the study of [1] and results from STAR CCM+ model for validation purposes. Run 112 specifications: $T_{0}=42.2^{\circ} \mathrm{C}, p_{0}=55.9 \mathrm{bar}, p_{\max }=192.4 \mathrm{bar}$. Run 213 specifications: $T_{0}=3.9^{\circ} \mathrm{C}, p_{0}=47.8 \mathrm{bar}, p_{\max }=223.8 \mathrm{bar}$. Run 311 specifications: $T_{0}=61.9^{\circ} \mathrm{C}, p_{0}=59.5 \mathrm{bar}, p_{\max }=158.3 \mathrm{bar}$.

\section{Numerical Results and Comparison}

For this section, a compression-expansion case is studied which is based on the initial conditions of the run 233 described in Table 1. The objective is to illustrate the dynamics of the system. The simulation is initialized with a temperature of $33.6^{\circ} \mathrm{C}$ and a pre-charge pressure of $50 \mathrm{bar}$, with an initial volume of 5.21 liters and a compression ratio of 2.7. Figure 14a shows the volume of the gas chamber during the process. The accumulator is oriented vertically.

For the CFD model, the reference signal of the volume changes in time were used. Unusual behavior of the heat transfer in the system was experienced when the signal inputed into the CFD model had some level of noise (when, for example, the measured piston position from the experiments was used as an input to the piston position in the CFD model). Large amounts of heat could be seen transfered from the gas to the walls of the accumulator. This 
sensitivity can be a consequence of the choice of the turbulence model selected. The $k-\epsilon$ turbulence model assumes that turbulent stability is achieved, and the noise in the piston movement can excite the system enough to create a highly turbulent behavior near the walls, causing excessive heat loss and unreal results.

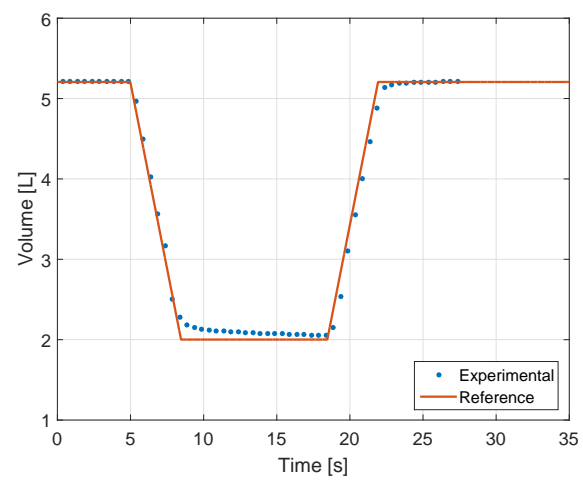

(a) Gas Volume

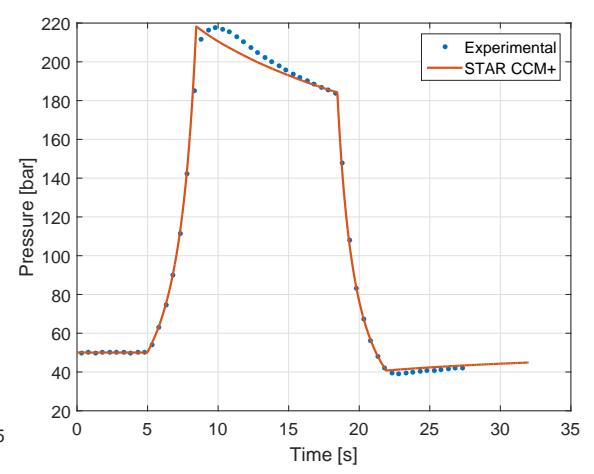

(b) Gas Pressure

Figure 14: Gas volume and pressure for the compression-expansion cycle

The pressure evolution obtained by the CFD simulation has a good level of agreement with the experimental reference, Figure 14b, which provides a solid validation point for the model. The discrepancies may originate from the way the position of the piston is controlled in the experimental setup, which is equivalent as filtering the input signal. This smoothing is omitted in the CFD model due to the fact that it would require the measured piston position from the experiments, which contains noise and can produce unreliable results due to the issues explained above.

\subsection{Temperature Distribution}

Figure 15 shows the evolution of the temperature distribution in the accumulator for the compression-expansion cycle. The compression process starts at $\mathrm{t}=2.0$ seconds, the gas increases temperature due to the reduction of the volume, however the regions close to the cylinder shell remain at lower temperatures due to the heat exchange with colder solid domain. At $\mathrm{t}=4.0$ seconds, a vortex of colder gas can be seen emerging from the piston, revolving clockwise towards the walls of the accumulator. The movement of the piston drags the colder gas in the boundary layer at the inner wall of the cylinder, and is pushed inwards into the centerline of the domain, passing above the piston. From $t=6.0$ seconds to $t=14.0$ seconds the compression is held. The gas reaches a maximum temperature of $184{ }^{\circ} \mathrm{C}$, which decreases over time due to the diffusion and natural convection. It can be seen that the temperature is clearly stratified, from top to bottom of the gas domain. Temperature differences between the top of the gas domain and the bottom (where the piston lies) of up to $60^{\circ} \mathrm{C}$ are visible. The solid parts of the accumulator surrounding the gas domain also increase their temperature, up to $10{ }^{\circ} \mathrm{C}$, due to heat transfer from the hotter gas. At $\mathrm{t}=16.0$ seconds, the expansion process has begun. The gas temperature near the piston decreases faster than the temperature at the top, as the gas expands faster due to the influence of the piston motion. Clear temperature gradients can be seen along the domain in the vertical direction as in the compression part. Now the heat transfer process is reversed, the solid parts of the accumulator are at a higher temperature than the gas. The shell of the accumulator remains at a higher temperature in the areas where the volume was held after the compression.

The results show that temperature gradients along the gas domain exist. Experimental temperature measurements of the gas are influenced by the position of the sensor. A set of virtual probes were setup in the CFD model to compare with the experimental measurements. Figure 16 shows the temperature measurements at different positions in the gas domain. As shown in the aforementioned results, the temperatures measured at the top of the domain are higher than the ones on the piston, due to the gradient imposed by the gravitational forces. Simulated results of the temperatures in the probes have fluctuations, a consequence of the fluid dynamics in the gas, when hotter and colder gas move near the probe. The simulated bulk (or average) temperature of the domain, calculated as the mass averaged temperature of the gas, lies in between the top and the piston temperatures, as it behaves as an average expression of the temperature in the whole domain. Experimental results show very good agreement with the simulated ones, and 

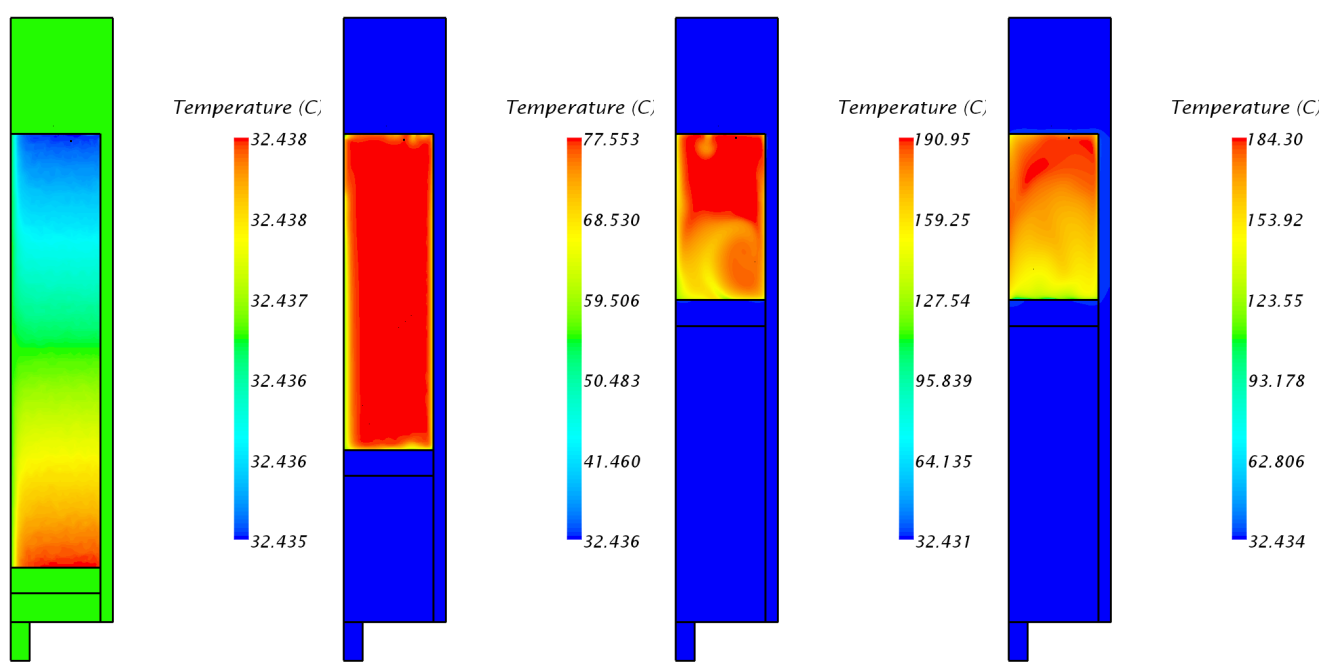

(a) $\mathrm{t}=0.0 \mathrm{~s}$

(b) $\mathrm{t}=2.0 \mathrm{~s}$

(c) $\mathrm{t}=4.0 \mathrm{~s}$

(d) $\mathrm{t}=6.0 \mathrm{~s}$
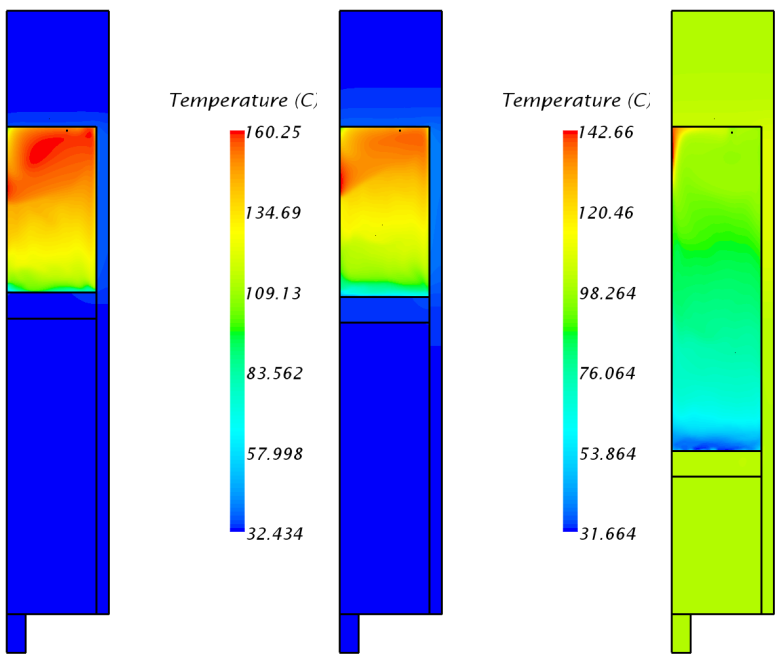

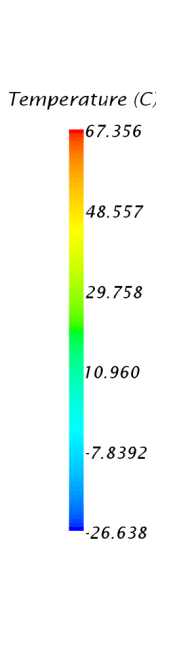

(g) $\mathrm{t}=16.0 \mathrm{~s}$

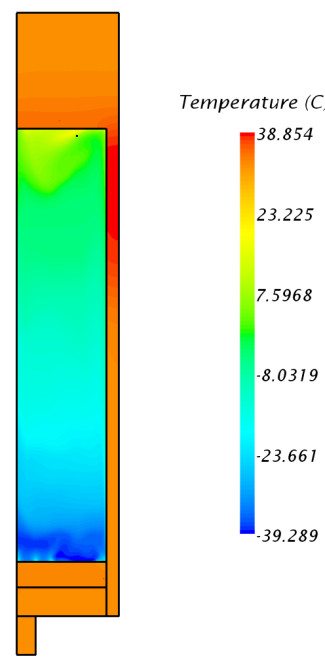

(h) $\mathrm{t}=18.0 \mathrm{~s}$

(e) $\mathrm{t}=10.0 \mathrm{~s}$

(f) $\mathrm{t}=14.0 \mathrm{~s}$

Figure 15: Temperature distribution in the accumulator during the compression-expansion cycle

reflect the importance of choosing an appropriate measuring position of the temperature sensors in the experimental setup to have a good reference to compare simulation models. Measured temperatures from the top of the domain provide a good approximation to the average temperature of the gas. Measurements of the outer temperature of the shell were also monitored at specific points (Figure 16b). The simulation results show a similar behavior to the experimental ones. Small discrepancies can be due to isolation problems in the temperature probes of the experimental setup or changes in the environmental conditions (higher air velocities or lower temperatures).

Figure 17 shows the evolution of the velocity field during the compression, near the piston. The movement of the piston induces momentum into the fluid, dragging the colder gas close to the boundary layer near the cylinder wall into the piston. This generates high fluid velocities above the piston with fluid moving upwards the domain. Gas from the centerline of the accumulator is also dragged into the piston, encountering itself with the other gas stream from the cylinder walls. This results in the formation of clear vortices above the piston at $t=8.5$ seconds. The movement of gas upwards generates a clockwise swirling motion where the gas experiences also high downwards velocities near the cylinder's walls. 


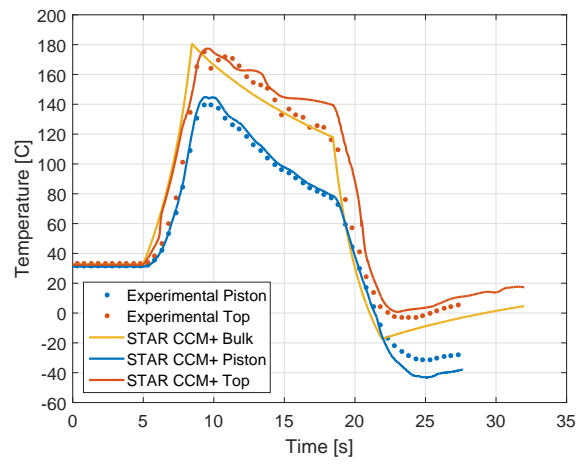

(a) Gas Domain Temperatures

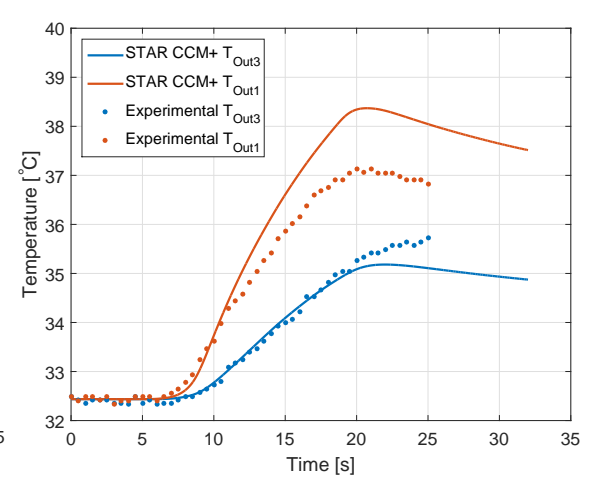

(b) Outer Shell Temperatures

Figure 16: Temperature measurements in the accumulator. Experimental vs. CFD results

Figure 18 shows the flow movement due to natural convection, after the compression phase finalizes. The hot gas close to the solid parts experiences fast heat transfer with the cold boundaries and its temperature drops (Figure 18b). Consequently, the density of this colder gas increases, and it experiences high velocities downwards. This can be seen in Figure 18a, where high velocity gradients and magnitude in the vertical direction are present close to the cylinder wall. Visible vortices can also be spotted in the gas due to natural convection. Small scale vortices can be seen above the piston, in the bottom left corner of the domain. Higher scale vortices form at the top of the domain, where high temperature gas is located. Near the cylinder walls, vertical lines can be seen, describing the downwards movement of the cold gas.

\subsection{Heat Transfer Across Boundaries}

With the CFD model, the boundary fluxes in the walls of the gas domain can be integrated to obtain the heat transfered between bodies. The resulting rate of heat losses are presented in Figure 19a. It can be seen that the heat losses follow the dynamics of the compression-expansion cycle. Heat loss increases as the gas is compressed due to the continual increase of temperature and the momentum induced in the gas due to the piston movement. After the compression, the heat transfer gradually decays due to a sustained decline of the gas temperature and the increase of temperature in the solid bodies of the accumulator. During the expansion, the heat transfer decreases gradually as the gas gets colder. At around 20 seconds into the simulation the heat transfer process reverses, now the gas receives heat from the hotter boundaries. When the expansion finalizes, the gas keeps receiving heat from the solid boundaries, and the magnitude of the heat transfer diminishes as time passes, due to the gas and the solid boundaries reaching slowly thermal equilibrium. Most of the heat is transfered through the cylinder shell boundaries. This responds to the fact that it has the biggest heat transfer area (which is between 10 and 2 times higher than the one of the piston or the top boundary) and high fluid velocities because of natural convection effects (as seen in Figure 18a). The heat losses through the piston and top boundary are smaller, accounting $15 \%$ of the total. It can be seen that for the top boundary the heat transfer process does not experience an inversion when the expansion begins. This is due to the small temperature difference between the gas and the boundary. In the piston, a small recovery can be seen after the compression. The lowest temperatures occur near this boundary so the temperature difference is high enough to be able to recover some of the heat.

The heat transfer and the corresponding heat transfer coefficients, associated to each boundary, (Figure 19a and 19b) show that after the compression, the highest heat transfer is experienced in the cylinder wall, while the lowest is in the piston. The temperature difference between the piston and the cold gas at the bottom of the domain is the smallest between the boundaries, which results in lower heat transfer. On the top boundary, the higher gas temperatures are found, which increases the heat transfer coefficient. After the expansion, the piston-gas interface has the highest values of $h$, a consequence of the higher temperature difference between the now colder gas at the bottom of the gas domain and the piston. Still, although having a reduced heat transfer coefficient, the highest amount of heat is transfered through the cylinder walls due to the large heat transfer area. 


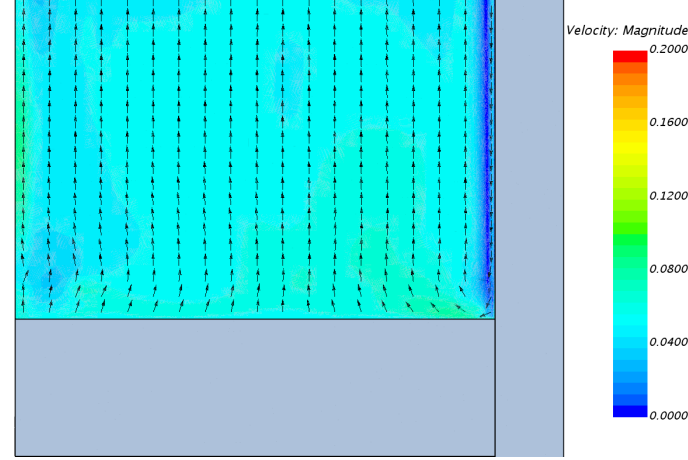

(a) $\mathrm{t}=6.5 \mathrm{~s}$

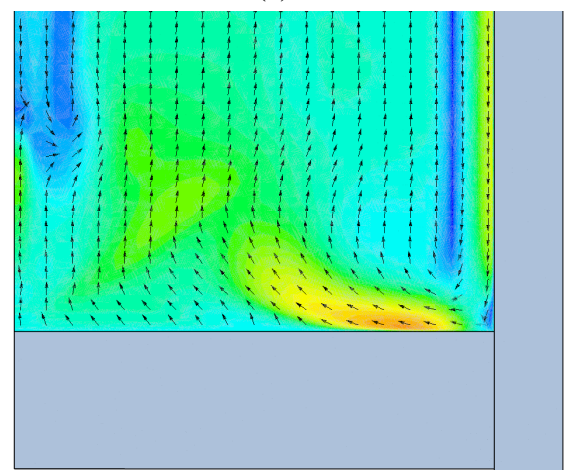

(c) $\mathrm{t}=7.5 \mathrm{~s}$

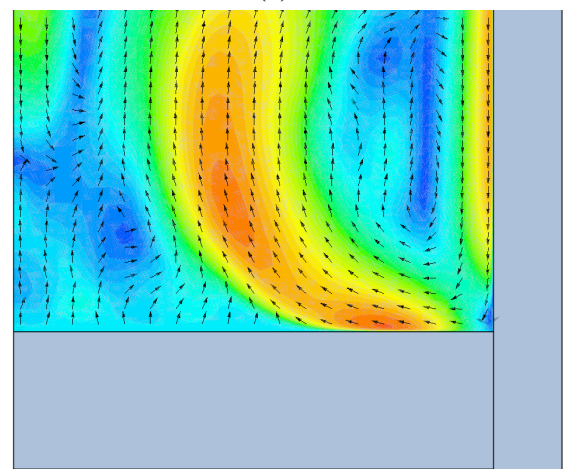

(e) $\mathrm{t}=8.5 \mathrm{~s}$

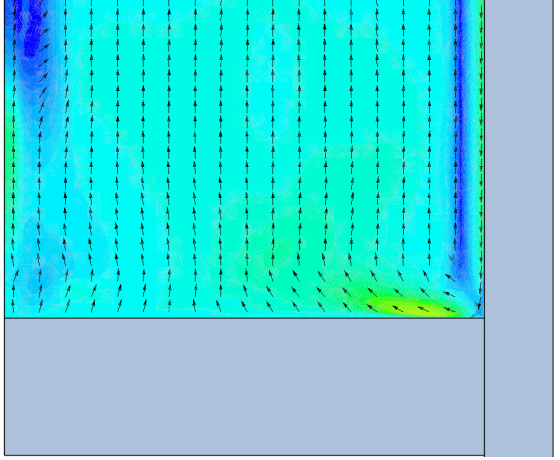

(b) $\mathrm{t}=7.0 \mathrm{~s}$

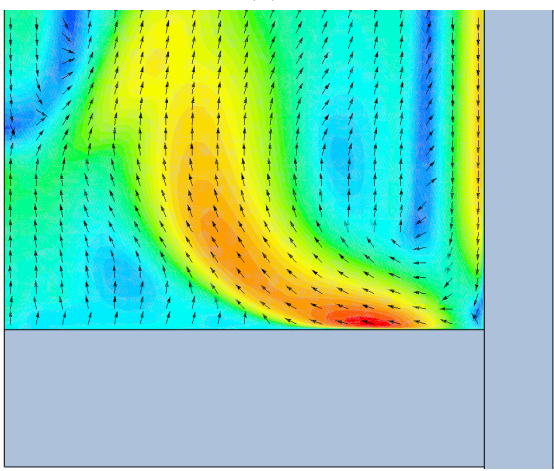

(d) $\mathrm{t}=8.0 \mathrm{~s}$

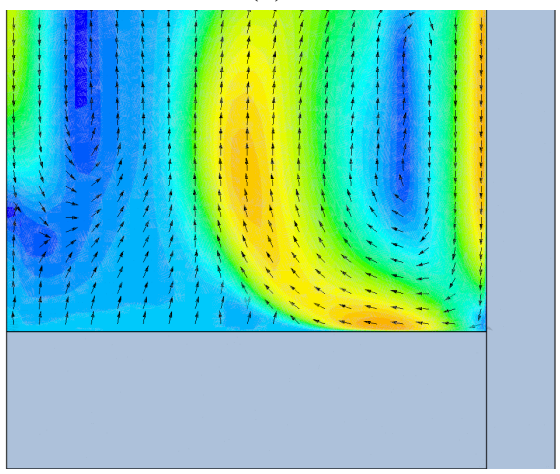

(f) $t=9.0 \mathrm{~s}$

Figure 17: Vectorial representation of the velocity field of the gas domain near the piston during compression. The shown area corresponds to a height of $20 \mathrm{~cm}$ over the piston into the gas side.

The curves represented in Figure 19b suggest that there is a clear non linear behavior of the heat transfer coefficient to the temperature difference between the gas and the surroundings. This implication has a direct influence in the way the heat losses are linearly modeled in Equation 11, and specifically on how $\tau$ is conceived mathematically. Non linearities arise due to the fact that the fluid flow models are very non linear (incompressible Navier-Stokes, turbulence modeling), and the thermodynamical behavior of the system is influenced by the momentum of the flow and the local changes of viscosity and density, which themselves are non linear functions of the temperature.

\subsection{The Thermal Time Constant}

The dynamic behavior of the heat transfer phenomena in the gas domain can be expected to be reflected in the thermal time constant of the gas. Figure 20 shows the variations of the thermal time constant of the gas over time. 


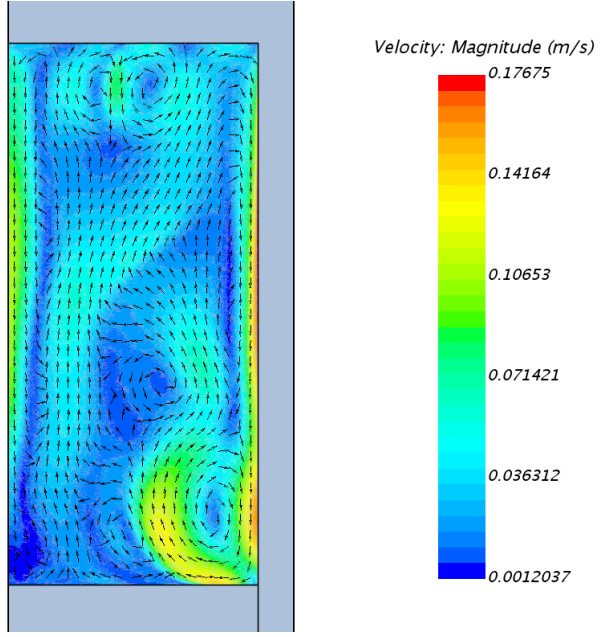

(a) Velocity Magnitude

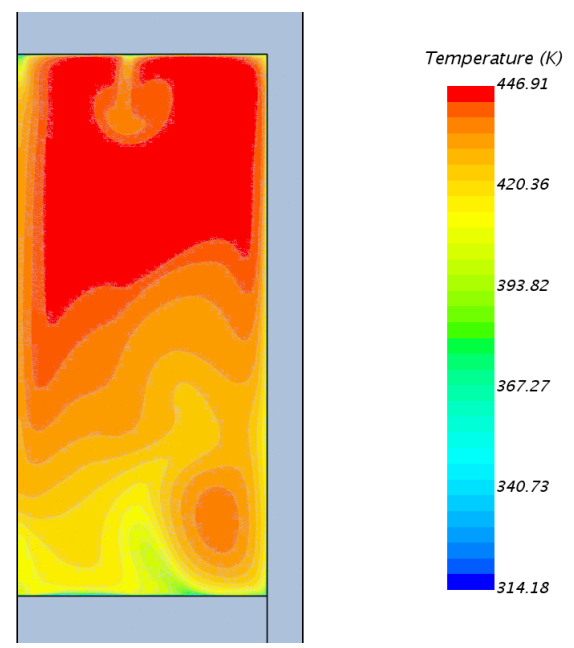

(b) Temperature

Figure 18: Velocity field and Temperature of the gas domain after compression $(\mathrm{t}=10$ seconds $)$

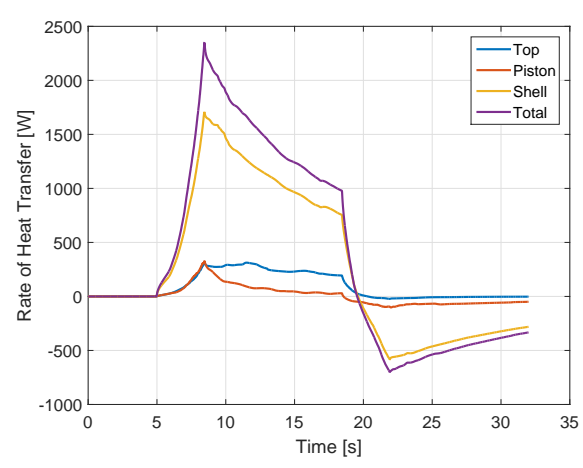

(a) Gas Domain Heat Loses

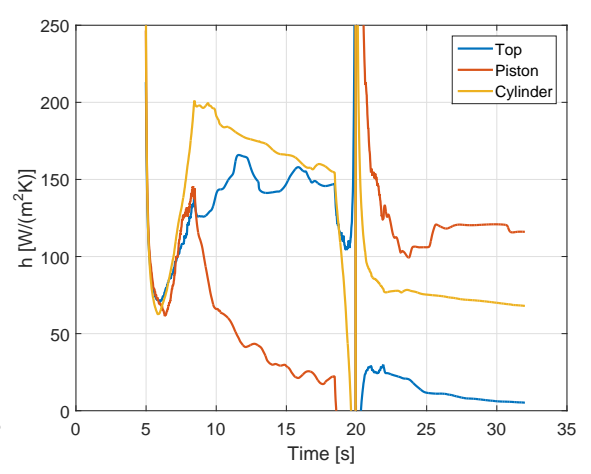

(b) Heat Transfer Coefficients

Figure 19: Boundary heat losses in the gas domain from the CFD simulation of the case studied.

Initially, as the process is initialized, the thermal time constant has a value of zero. This is due to the way the thermal time constant is mathematically defined to be calculated in the simulation. $\tau$ can be defined by combining Equation 13 and Equation 4 as

$$
\tau=\frac{m C_{V}\left(T_{s}-T\right)}{Q}
$$

where $Q$ is the heat loss across the boundaries of the gas domain and $T_{s}$ is the temperature of the walls (which now replaces $T_{a}$ since the gas in reality exchanges heat with the cylinder walls, and the temperature of these walls changes over time). When the compression starts, $\tau$ increases, and reaches a maximum value, and afterwards starts decreasing to its lowest value at the end of the compression. This behavior can be explained by looking at the way the temperature and the heat losses evolve in time, shown in Figure 21. It can be seen that at the beginning of the compression, the rate of heat transfer grows slower than the temperature difference between the gas and the boundaries. At 6 seconds into the simulation, the rate of heat transfer starts growing faster until at 7 seconds it has the same rate of change as the temperature difference. This increase in the heat transfer dynamics can be explained by the velocity that is induced into the gas due to the movement of the piston. Recalling Figure 17, during compression, high velocities are developed in the fluid close to the piston due to the dragging effect of its sliding motion in the cylinder boundaries, and this effect generates swirling motions in the gas chamber. The beginning of this effect starts at 6 seconds into the simulation, where velocities start developing near the piston and cylinder walls. This coincides with the peak of the 
$\tau$ constant during compression. This shows that the convective heat transfer is activated by the piston motion. The gas temperature increases proportionally to the compression speed. After the fluid is set in motion by the piston, the convective heat transfer is started and consequently $\tau$ decreases until it reaches a minimum value at the end of the compression phase.

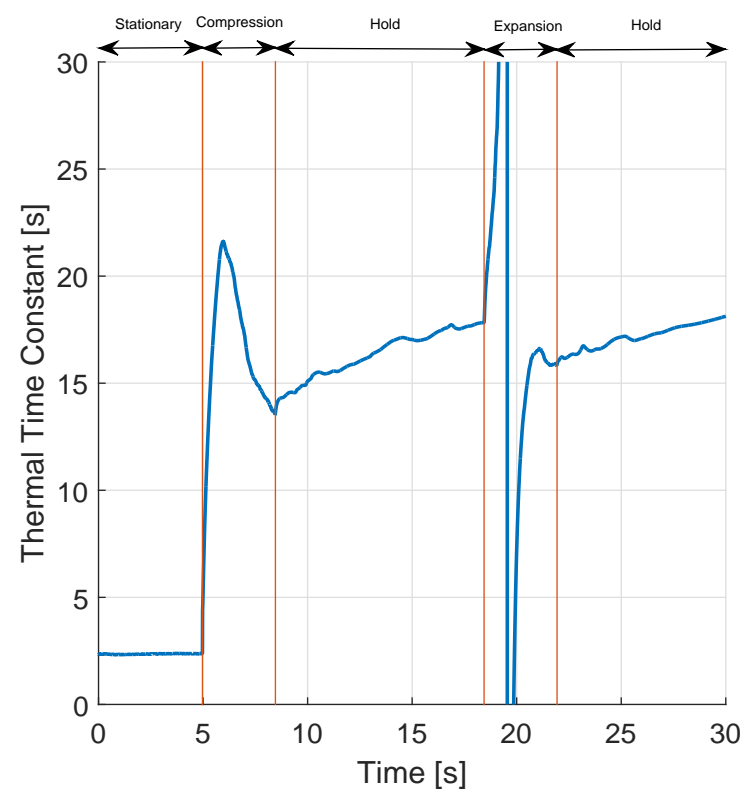

Figure 20: Thermal time constant evolution for a compression-expansion cycle

When the compressed gas is held, $\tau$ increases slowly, almost linearly. This corresponds to the steady heat transfer between the gas and the surrounding boundaries, which are slowly reaching thermal equilibrium. This behavior can also be seen after the expansion when the volume remains constant. During the expansion, the value of $\tau$ jumps from positive to negative suddenly. This is a result of how the time constant is defined mathematically. The denominator of Equation 17 is close to zero when there is no heat transfer, resulting in a visible discontinuity of the thermal time constant. This is a direct consequence of the temperature of the gas reaching lower temperatures than the boundaries due to the dilation of the chamber, and instantaneously the temperature difference is zero, thus also the heat exchange. The large value of the time constant, now with sign changed, is a consequence of the heat transfer and direction of motion being out of phase which is also discussed by Catto[8].

It can be seen in Figure 21 in the detailed view of the expansion that the heat transfer reaches zero value first than the temperature difference. The heat transfer slows down when the expansion is finishing, and changes direction, such that heat is being transfered from the solid bodies to the gas. The temperature differences between the gas and the boundaries now peaks at $50{ }^{\circ} \mathrm{C}$, which is 3 times smaller than the maximum temperature difference in compression. This results in a decreased heat transfer. Also, the low temperatures increase the density and viscosity of the gas, diminishing its heat transfer coefficient.

\section{Conclusions}

The thermodynamical processes involved in a hydraulic accumulator during operation were successfully studied by the use of a CFD model. The results obtained from this model show a high level of agreement with the experimental results for a range of cases. The results demonstrate that the heat losses in the gas side of an accumulator are mainly associated with heat transfered to the solid parts. The heat losses to the surroundings are relatively small for the conditions considered and have no significant effect in the system. There is also clear evidence that the temperature is stratified such that the heat losses are non-uniform throughout the boundaries. 

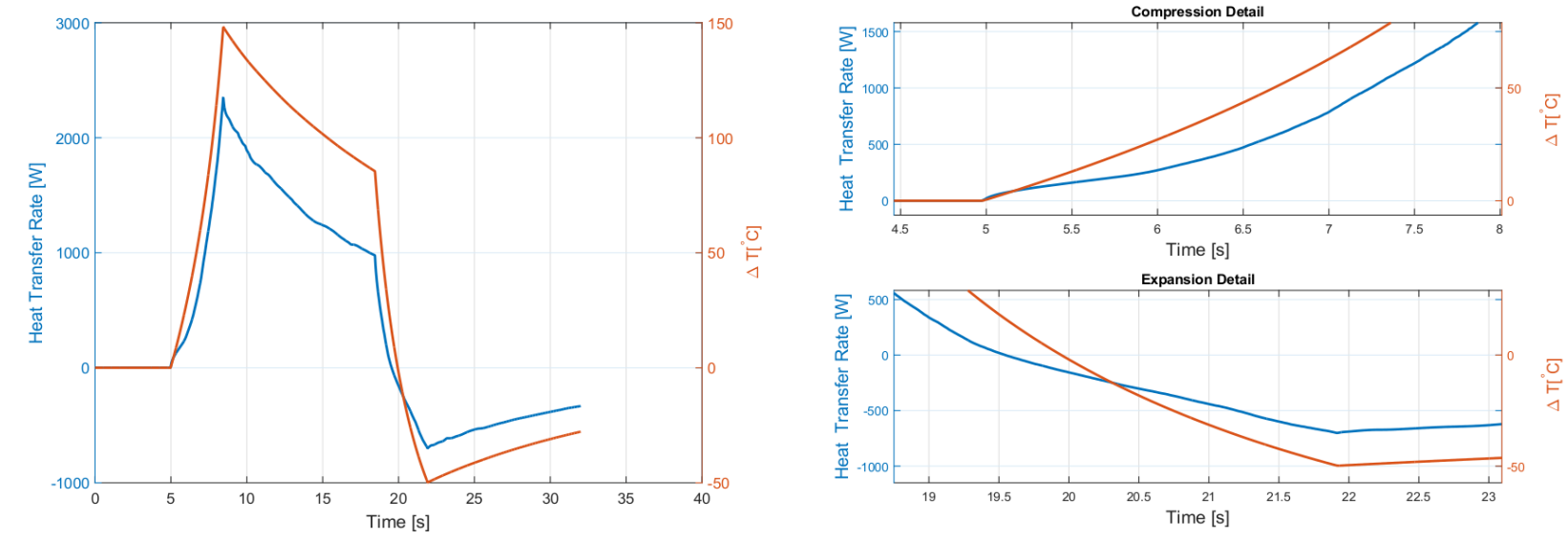

Figure 21: Evolution in time of the heat losses and temperature difference between the gas and the boundaries

It has furthermore been shown that the thermal time constant is indeed not constant over time but varies due to the dynamics of the gas flow induced by the piston movement. The non-linear behavior of the heat transfer coefficient and thereby thermal time constant are generated by the fluid motion and the convective processes. By the use of the CFD model consistent and more precise results compared to the thermal time constant one dimensional models have been obtained. The inclusion the proper fluid and thermodynamics in the CFD model, as well as the turbulent motion of the fluid, provides consistent results within $10 \%$ of the measured experimental results.

The limitations of the proposed CFD model of the paper rely on the simplified conception of a 2D model. A full 3D model of the accumulator including the geometrical details omitted may improve the predictions; however, the increased insight into the physics of course comes at a significant computational cost. With a 3D model other influencing effects such as the rotation of a hub mounted accumulator could also be studied. The thermal time constant model is simple to implement and with the right values of the thermal time constant, provides good levels of accuracy. This paper successful suggests to obtain these through experiments and identification; however, this requires that the operating conditions are known to a certain extend.

Future investigations could very well be focused on increasing this time constant of the gas and reduce the heat losses. The inclusion of foams [3] or heat sinks [18] in the gas chamber are proving to be efficient for this purpose. From these ideas, several studies regarding material, shape and positioning in the gas chamber can be derived and, supported with appropriate CFD models and experimental data using the presented setup.

\section{Acknowledgments}

This project was funded and supported by Fritz Schur Energy A/S (FSE), in Glostrup, Denmark, by providing the hardware, software as well as the technical knowledge to help fulfill the investigation.

\section{References}

\section{Appendix A. Long compression hold-cycle}

Figure A.22 confirms the first order behavior of the system which can be difficult to confirm at e.g. figure 8 and thus qualify the usage of a thermal time constant model.

[1] H. Hansen, P. Rasmussen, Modelling hydraulic accumulators for use in wind turbines, Proceedings of the 13th Scandinavian International Conference on Fluid Power Linköping, Sweden.

[2] D. R. Otis, Predicting performance of gas charged accumulators, Proceedings of the 1st Fluid Power and Control Systems Conference University of Wisconsin-Madison.

[3] A. Pourmovahed, N. Beachley, F. Fronczak, Modeling of an hydraulic energy regeneration system - part i: Analytical treatment, Transactions of the ASME 114 (1992) 160-165. 

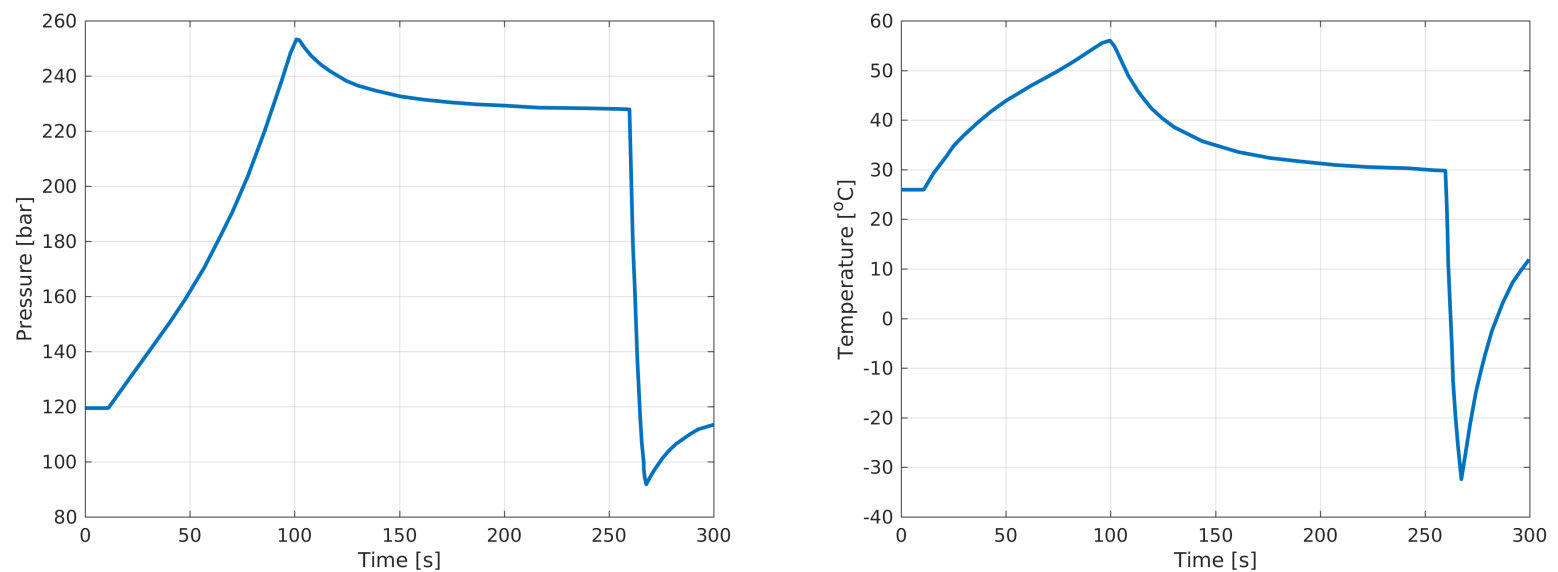

Figure A.22: 90 seconds compression-hold-expansion cycle for the accumulator with preload pressure $120 \mathrm{bar}$ at $20^{\circ} \mathrm{C}$ to confirm the first order behavior of the system.

[4] S. Rotthäuser, Verfahren zur Berechnung und Untersuchung hydropneumatischer Speicher, Fakultät für Maschinenwesen der RheinischWestfälische Technische Hochschule Aachen, 1993.

[5] K. Rupprecht, Hydrospeicher, Experimentelle und analytische Untersuchungen zur Energiespeicherung, Fakultät für Maschinenwesen der Rheinisch-Westflische Technische Hochschule Aachen, 1988.

[6] J. Svoboda, G. Bouchard, S. Katz, A thermal model for gas-charged accumulators based on the heat conduction distribution, Fluid Transients and Acoustics in the Power Industry (1978) 161-167.

[7] H. Faulkner, J. Smith, Instantaneous heat transfer during compression and expansion in reciprocating gas handling machinery, Proceedings 18th IECEC (1983) 724-730.

[8] A. G. Catto, A. T. Prata, A numerical study of instantaneous heat transfer during compression and expansion in piston-cylinder geometry, Numerical Heat Transfer, Part A: Applications 38 (3) (2000) 281-303. doi:10.1080/10407780050136530.

[9] M. Ota, Y. Haramura, Heat Transfer During Compression and Expansion of Gas, Journal of Heat Transfer 130 (3) (2008) 032801. doi:10.1115/1.2804949.

[10] P. Puddu, M. Paderi, Hydro-pneumatic accumulators for vehicles kinetic energy storage: Influence of gas compressibility and thermal losses on storage capability, Energy 57 (2013) 326-335. doi:10.1016/j.energy.2013.04.072.

[11] A. Pfeffer, T. Glück, W. Kemmetm?ller, A. Kugi, Mathematical modelling of a hydraulic accumulator for hydraulic hybrid drives, Mathematical and Computer Modelling of Dynamical Systems 22 (5) (2016) 397-411. doi:10.1080/13873954.2016.1174716.

[12] D. R. Otis, A. Pourmovahed, An algorithm for computing nonflow gas processes in gas springs and hydropneumatic accumulators, ASME Journal of Dynamic Systems 107 (1985) 93-96.

[13] D. R. Otis, A. Pourmovahed, An experimental thermal time-constant correlation for hydraulic accumulators, Journal of Dynamic Systems, Measurement and Control 112 (1990) 116-121.

[14] G. A. F. Seber, C. J. Wild, Nonlinear Regression, Hoboken, NJ. Wiley-Interscience, 2003.

[15] F. Incropera, D. DeWitt, A. Lavine, T. Bergman, Fundamentals of Heat and Mass Transfer, John Wiley and Sons, 2011.

[16] J. Bardina, P. Huang, T. Coakley, Turbulence modeling validation, testing, and development, NASA Technical Memorandum 110446.

[17] B. M. Limited, Hyspin awh-m range oils. anti-wear hydraulic oils., uS Patent 2,331,921 (Oct. 19 2009). URL http://msdspds. castrol. com/bpglis/

[18] J. Juhala, J. Kajaste, M. Pietola, Improving energy efficiency of hydraulic accumulators, Proceedings of the 12th Scandinavian International Conference on Fluid Power Tampere, Finland. 